Pure and Applied Mathematics Quarterly

Volume 1, Number 1, 28-67, 2005

\title{
Shafarevich's Conjecture for CY Manifolds I
}

\author{
Kefeng Liu, Andrey Todorov \\ Shing-Tung Yau and Kang Zuo
}

\begin{abstract}
Let $C$ be a Riemann surface with fixed finite number of points on it. Given a Calabi-Yau (CY) manifold with fixed topological type, we consider the set of all families of CY manifolds of fixed topological type over $C$ with degenerate fibres over the fixed points up to isomorphism. This set is called the Shafarevich set. The analogue of Shafarevich conjecture for CY manifolds is for which topological types of CY the Shafarevich set is finite. We relate the analogue of Shafarevich problem for CY manifolds to the non-vanishing of the Yukawa coupling. We also study in this paper some moduli problems related to the Shafarevich conjecture. We also give a counter example of the Shafarevich problem for a class of CY manifolds.
\end{abstract}

\section{Contents}

1 Introduction

2 Moduli of Polarized CY Manifolds

2.1 Construction of the Teichmüller Space of Marked Polarized CY Manifolds . . . . . . . . . . . . . . . . . . . 32

2.2 Construction of the Moduli Space of Polarized CY Manifolds 39

Received December 8, 2004. 
3 Moduli of Maps $\quad 41$

3.1 Smoothness of the Moduli Space of Maps . . . . . . . . . . 41

3.2 Some Applications . . . . . . . . . . . . . . 44

4 Yukawa Coupling and Rigidity 45

5 Yau's Form of Schwartz Lemma and Boundedness 49

6 Higgs Bundles, VHS and Rigidity 51

7 Appendix I. Complex Exponential Maps in Kähler Geometry

8 Appendix II. Applications of Bishop's Convergence Theorem

9 Appendix III. Counter Examples to the Analogue of Shafarevich Conjecture of CY Manifolds

\section{Introduction}

Let $\mathrm{C}$ be a fixed, non-singular algebraic curve, and let $\mathrm{E}$ be a fixed effective divisor on $\mathrm{C}$ such that all the points in $\mathrm{E}$ have multiplicity 1. Define $\mathrm{Sh}(\mathrm{C}, \mathrm{E}, \mathrm{Z})$ to be the set of all isomorphism classes of projective varieties $\pi: \mathcal{Z} \rightarrow C$ with a fibre of "type" $Z$ such that the only singular fibres are over the set E. The general Shafarevich type problem is: "For which "type" of varieties $Z$ and data $(C, E)$, is $S h(C, E, Z)$ finite?

In his article published in the Proceedings of the International Congress of Mathematicians, Stockholm meeting held in 1962, Shafarevich wrote:"One of the main theorems on algebraic numbers connected with the concept of discriminant is Hermit's theorem, which states that the number of extensions $k^{\prime} / k$ of a given degree and given discriminant is finite. This theorem may be formulated as follows: the number of extensions $k^{\prime} / k$ of a given degree whose critical prime divisors belong to a given finite set $S$ is finite." Inspired by this result of Hermit, Shafarevich conjectured in [37]: "There exists only a finite number of fields of algebraic functions $K / k$ of a given genus $g \geq 1$, the critical prime divisors of which belong to a given finite set $S$." In an unpublished work, Shafarevich proved his conjecture in the setting of hyperelliptic curves.

In the case when $Z$ is a curve of genus $g>1$ and $\mathrm{E}$ is empty, Parshin proved in [29] that $\mathrm{Sh}(\mathrm{C}, \mathrm{E}, \mathrm{Z})$ is finite, and Arakelov proved in [2] the 
finiteness in the case $\mathrm{E}$ is not empty. Faltings constructed examples showing that $\mathrm{Sh}(\mathrm{C}, \mathrm{E}, \mathrm{Z})$ is infinite for abelian varieties of dimension $\geq 8$. See [13]. Saito an Zucker extended the construction of Faltings to the setting when $\mathrm{Z}$ is an algebraic polarized K3 surface. They were able to classify all cases when the set Sh $(\mathrm{C}, \mathrm{E}, \mathrm{Z})$ is infinite. See [33]. Faltings proved the Shafarevich's conjecture over number fields and thus he proved the Mordell conjecture. Yves Andre proved the analogue of Shafarevich's conjectures over the number fields for K3 surfaces. See [1]. Using techniques from harmonic maps Jost and Yau analyzed Sh (C,E,Z) for a large class of varieties. See [20]. Ch. Peters studied finiteness theorems by considering variations of Hodge structures and utilizing differential geometric aspects of the period map and the associated metrics on the period domain. See [30].

A. Parshin and E. Bedulev have proved the boundedness for families of algebraic surfaces over a fixed algebraic curve assuming that all the fibres are non-singular. E. Bedulev and E. Viehweg have proved the boundedness for families of algebraic surfaces over a fixed algebraic curve, which possibly have singular fibres. See [7]. Migliorni, Kovác and Zhang proved that any family of minimal algebraic surfaces of general type over a curve of genus $\mathrm{g}$ and $\mathrm{m}$ singular points such that $2 \mathrm{~g}-2+\mathrm{m} \leq 0$ is isotrivial. See [21], [27], [48] and [7]. Recently very important results of Viehweg and the last named author appeared in [42], [43] and [44]. Brody hyperbolicity was proved for the moduli space of canonically polarized complex manifolds. In [44] 6.2 a) They proved the boundedness for $\operatorname{Sh}(\mathrm{C}, \mathrm{E}, \mathrm{Z})$ for arbitrary $Z$, with $\omega_{Z}$ semi-ample. They also established that the automorphism group of moduli stacks of polarized manifolds is finite. The rigidity property for the generic family of polarized manifolds was also proved. The basis idea in the proof is to consider the non-vanishing property of the maximal iterated Kodaira-Spencer map. One notices that in the case of Calabi-Yau manifolds this maximal iterated Kodaira-Spencer map is related to the Yukawa coupling. In this paper the relation between the Yukawa coupling and rigidity problems for $\mathrm{CY}$ manifolds is first formally formulated.

We are going to study the analogue of the Shafarevich's conjecture about finiteness of the families of polarized CY manifolds over a fixed Riemann surface with prescribed points of degenerate fibres. Any family of polarized CY manifolds induces a map from the base without discriminant locus to the coarse moduli space. Thus Shafarevich conjecture is reduced to prove that moduli space of maps of Riemann surface into the moduli space of polarized CY manifolds with some additional properties is a finite set. It is not difficult to show that the existence of Kähler metric with negative bounded holomorphic curvature and the rigidity of any family of CY 
manifolds over a Riemann surface with fixed points of degenerations, Yau's form of Schwarz Lemma and the Bishop compactness imply the Shafarevich conjecture. We used the Yukawa coupling to check rigidity:

Condition 1 Suppose that

$$
\pi: \mathcal{X} \rightarrow C
$$

is a family of polarized $C Y$ manifolds over a Riemann surface $C$. Let $t_{0} \in C$ such that for $M=\pi^{-1}\left(t_{0}\right)$ the following condition is satisfied: for any non zero $\phi \in H^{1}\left(M, T^{1,0} M\right), \wedge^{n} \phi \neq 0$ in $H^{n}\left(M,\left(\Omega_{M}^{n}\right)^{*}\right)$. Then the family (1) is rigid.

The condition 1 brings up an interesting relation between rigidity and mirror symmetry. We would also like to compare Condition 1 with some of the results obtained in [43]. In [45] some important applications of the Condition 1 are obtained. In [45] the finiteness of those families, whose iterated Kodaira-Spencer maps have the same length as the length of the iterated Kodaira-Spencer map of the moduli space, are obtained. E. Viehweg and the last named author constructed in [46] rigid families of CY hypersurfaces for which the condition 1 does not hold, i.e. the Yukawa coupling is zero. They also have constructed non-rigid families of hypersurfaces in $\mathbb{C P}^{n}$ for any $n>2$ and of any degree $d>3$. See [46]. Recently Y. Zhang obtained some important results. More precisely he proved that any Lefshetz family of CY manifold is rigid. See [49] and [50].

In this paper our approach is close to that of Jost and Yau. Instead of harmonic maps in this paper we are using holomorphic ones. See [20]. Our paper is based on the study of the moduli space of polarized CY manifolds and its Teichmüller space. We will show that the Teichmüller space $\widetilde{T}(\mathrm{M})$ of a Calabi-Yau manifold $\mathrm{M}$ exists and it has finite number of irreducible components. Each component $\mathcal{T}(\mathrm{M})$ of $\widetilde{T}(\mathrm{M})$ is a non-singular complex manifold. Moreover over $\widetilde{T}(\mathrm{M})$ there exists a universal family of marked polarized CY manifolds up to the action of a finite group of complex analytic automorphisms which acts trivially on the middle cohomology.

The existence of the coarse moduli space $\mathfrak{M}_{L}(\mathrm{M})$ of polarized CY manifolds as a quasi-projective variety was proved by Viehweg. See [41]. Yau conjectured that Mumford-Chow stability of the canonically embedded projective manifolds is equivalent to the existence of Kähler-Einstein metric. Recently Donaldson proved that the existence of constant scalar curvature implies Chow stability. By combining the result of Donaldson with the solution of the Calabi conjecture given by Yau, one can reprove the result of Viehweg. 
In this paper we will prove the existence of a finite cover $\mathcal{M}_{L}(\mathrm{M})$ of the coarse moduli space $\mathfrak{M}_{L}(\mathrm{M})$ of polarized CY manifolds such that $\mathcal{M}_{L}(\mathrm{M})$ is a non-singular quasi-projective variety and over $\mathcal{M}_{L}(\mathrm{M})$ there exists a family of polarized CY manifolds. The construction of $\mathcal{M}_{L}(\mathrm{M})$ and the family over it is based on the existence of family of CY manifolds over the Hilbert scheme. We will apply the results obtained in this paper to the analogue of the Shafarevich's conjecture by studying the moduli space of holomorphic maps from a fixed Riemann surface with prescribed points of degenerate fibres to the moduli space of polarized CY manifolds with the Hodge metric.

Acknowledgement 2 This work was finished at the Institute of Mathematical Sciences of the Chinese University of Hong Kong. We are grateful to the Institute of Mathematical Sciences of the Chinese University of Hong Kong for their support and encouragement. Special thanks to Yi Zhang for a careful reading of an earlier version of our paper. The first and the second author would like to thank Center of Mathematical Sciences of Zhejiang University where the final version of this paper was written. This work is partially supported by the Institute of Mathematical Sciences, CUHK. The last named author is also supported by grants from the Research Grants Council of Hong Kong Special Administrative Region, China (Project No. CUHK 4034/02P).

\section{Moduli of Polarized CY Manifolds}

\subsection{Construction of the Teichmüller Space of Marked Po- larized CY Manifolds}

Theorem 3 Let $\pi: \mathcal{X} \rightarrow \mathcal{K}$ be the Kuranishi family of a polarized $C Y$ manifold $M=\pi^{-1}\left(\tau_{0}\right), \tau_{0} \in \mathcal{K}$. Suppose that $G$ is a group of holomorphic automorphisms of $M$ such that $G$ acts trivially on $H_{n}(M, \mathbb{Z})$ and preserves the polarization. Then $G$ is a finite group of holomorphic automorphisms of all the fibres of the Kuranishi family.

Proof: Since $\mathrm{G}$ acts trivially on $H^{n}(\mathrm{M}, \mathbb{Z})$ and fixes the polarization class $L$ then the uniqueness the Calabi-Yau metric that corresponds to $L$ implies that the group $G$ is a group of isometries of the CY metric. Since on CY manifolds there does not exist global holomorphic vector fields, we can conclude that $G$ is a discrete subgroup of the orthogonal group. The compactness of the orthogonal group implies that $G$ is finite. The local Torelli Theorem implies that $\mathcal{K} \subset \mathbb{P}\left(H^{n}(\mathrm{M}, \mathbb{Z}) \otimes \mathbb{C}\right)$. So $\mathrm{G}$ acts on $\mathcal{K}$ and fixes 
the point $\tau_{0}$. Since $\mathrm{G}$ acts trivially on $H^{n}(\mathrm{M}, \mathbb{Z})$, then it will act trivially on $\mathcal{K}$. Next we are going to prove that if $g \in G$ is any element of $G$ then it acts as complex analytic automorphism on each $\mathrm{M}_{\tau}=\pi^{-1}(\tau)$ for any $\tau \in \mathcal{K}$. This means that $g^{*}\left(I_{\tau}\right)=I_{\tau}$, where $I_{\tau} \in C^{\infty}\left(\mathrm{M}, \operatorname{Hom}\left(T_{\mathrm{M}}^{*}, T_{\mathrm{M}}^{*}\right)\right)$, $I_{\tau}^{2}=-i d$ is the integrable complex structure operator that defines $\mathrm{M}_{\tau}$ for $\tau \in \mathcal{K} . T_{\mathrm{N}}^{*}$ is the cotangent $C^{\infty}$ real bundle. It is a well known fact that $I_{\tau}=\left(A_{\tau}\right)^{-1} \circ I_{0} \circ A_{\tau}$, where

$$
A_{\tau}=\left(\begin{array}{cc}
i d & \phi(\tau) \\
\phi(\tau) & i d
\end{array}\right)
$$

See [40]. Let us recall that if $\Theta_{M}$ is the holomorphic tangent bundle then

$$
\phi(\tau) \in C^{\infty}\left(\mathrm{M}, \operatorname{Hom}\left(\Omega_{\mathrm{M}}^{1,0}, \overline{\Omega_{\mathrm{M}}^{1,0}}\right)\right) \approx C^{\infty}\left(\mathrm{M}, \Theta_{\mathrm{M}} \otimes \Omega_{\mathrm{M}}^{0,1}\right)
$$

and $\phi(\tau)$ satisfies the equation that guarantees the integrability

$$
\bar{\partial} \phi(\tau)=\frac{1}{2}[\phi(\tau), \phi(\tau)] \text { and } \bar{\partial}^{*} \phi(\tau)=0 .
$$

of the complex structure operator $I_{\tau}$ defined above.For all the details see [40]. Here $\bar{\partial}^{*}$ means the conjugate of the operator $\bar{\partial}$ with respect to the Calabi-Yau metric corresponding to the polarization class $L$. If we prove that for each $g \in G$

$$
g^{*}(\phi(\tau))=\phi(\tau)
$$

then (3) implies $g^{*}\left(I_{\tau}\right)=I_{\tau}$ and so $\phi(\tau)$ is a complex analytic automorphism of $\mathrm{N}_{\tau}$.

Proof of (3): According to [40] if we fix a basis $\phi_{1}, \ldots, \phi_{N}$ of harmonic forms of $\mathbb{H}^{1}\left(\mathrm{M}, \Theta_{\mathrm{M}}\right)$ then the solution of the equations (2) are given by the power series

$$
\phi(\tau)=\sum_{i=1}^{N} \phi_{i} \tau^{i}+\sum_{i_{1}+\ldots+i_{N}=m>1} \phi_{i_{1}, \ldots, i_{N}}\left(\tau^{1}\right)^{i_{1}} \times \ldots \times\left(\tau^{N}\right)^{i_{N}}=\sum_{m=1}^{N} \phi_{[m]}(\tau),
$$

where

$$
\phi_{[m]}(\tau)=\sum_{i_{1}+\ldots+i_{N}=m} \phi_{i_{1}, \ldots, i_{N}}\left(\tau^{1}\right)^{i_{1}} \times \ldots \times\left(\tau^{N}\right)^{i_{N}}
$$

and $\phi(\tau)$ satisfies the recurrent relation:

$$
\phi(\tau)=\sum_{i=1}^{N} \phi_{i} \tau^{i}+\frac{1}{2}(\bar{\partial})^{*} \circ \mathbb{G}[\phi(\tau), \phi(\tau)] .
$$


See [40]. Here $\mathbb{G}$ is the Green operator associated to the Laplacian with respect to the $\mathrm{CY}$ metric associated to the polarization class $L$. Notice that since $G$ is the group of isometries of CY metric, for any $g \in G$ the Green operator will be invariant, i.e. $g^{*} \mathbb{G}=\mathbb{G}$. In $[40]$ it is proved that if $\phi \in \mathbb{H}^{1}\left(\mathrm{~N}, \Theta_{\mathrm{N}}\right)$ is a harmonic form with respect to the CY metric then $\phi\lrcorner \omega_{\mathrm{M}}$ will be a harmonic form of type $(n-1,1)$ This fact together with the fact that $G$ acts trivially on $H^{n}(\mathrm{M}, \mathbb{C})$ imply that the group acts trivially on $\mathbb{H}^{1}\left(\mathrm{M}, \Theta_{\mathrm{M}}\right)$. This implies that the linear term of (4) satisfies

$$
g^{*}\left(\phi_{[1]}(\tau)\right)=\phi_{[1]}(\tau) .
$$

The proof of the fact that $g^{*} \phi(\tau)=\phi(\tau)$ is done by induction on the homogeneity of the terms of the power series (4). Formula (6) shows that $\phi_{[1]}(\tau)$ is invariant under the action of $g$. Since for the higher order terms of (4) the relation (5) implies

$$
\phi_{[k]}=\sum_{p+q=k}\left[\phi_{[p]}(\tau), \phi_{[q]}(\tau)\right]
$$

and $g^{*}\left(\phi_{[p]}\right)=\phi_{[p]}$ for $p<k$ then we can conclude that $g^{*} \phi_{[k]}(\tau)=\phi_{[k]}(\tau)$ for any $k>0$. So the relation(3) is proved. Theorem 3 is proved.

Definition 4 The Teichmüller space $\mathcal{T}(M)$ of a compact complex manifold $M$ is defined as follows: $\mathcal{T}(M):=\mathcal{I}(M) /$ Dif $f_{0}(M)$, where

$$
\mathcal{I}(M):=\{\text { all integrable complex structures on } M\}
$$

and Diffo $(M)$ is the group of diffeomorphisms isotopic to identity. The group Diff $\left(M_{0}\right)$ acts on $\mathcal{I}(M)$ as follows: let $\phi \in D i f f_{0}(M)$ then $\phi$ acts on integrable complex structures on $M$ by pull-back, i.e. if $I \in C^{\infty}(M, \operatorname{Hom}(T(M), T(M))$, then we define $\phi\left(I_{\tau}\right)=\phi^{*}\left(I_{\tau}\right)$.

Definition 5 We will call a pair $\left(M ; \gamma_{1}, \ldots, \gamma_{b_{n}}\right)$ a marked $C Y$ manifold if $M$ is a $C Y$ manifold where $\left\{\gamma_{1}, \ldots, \gamma_{b_{n}}\right\}$ is a basis of $H_{n}(M, \mathbb{Z}) /$ Tor.

Remark 6 Let $\mathcal{K}$ be the Kuranishi space. It is easy to see that if we choose a basis of $H_{n}(M, \mathbb{Z}) /$ Tor in one of the fibres of the Kuranishi family $\pi: \mathcal{M} \rightarrow \mathcal{K}$ then all the fibres will be marked, since as a $C^{\infty}$ manifold $\mathcal{X}_{\mathcal{K}} \approx M \times \mathcal{K}$.

Next we are going to construct a universal family of polarized marked CY manifolds $\pi: \mathcal{U}_{L} \rightarrow \mathcal{T}_{L}(\mathrm{M})$ up to the action of the group of complex analytic automorphisms $G$ which acts trivially on $H^{2}(\mathrm{M}, \mathbb{Z})$ on the fibres. 
Theorem 7 There exists a family of marked polarized CY manifolds

$$
\pi: \mathcal{U}_{L} \rightarrow \mathcal{T}_{L}(M)
$$

with the following properties: $\boldsymbol{A} . \mathcal{T}_{L}(M)$ is a smooth manifolds of complex dimension $h^{n-1,1}$. B. The holomorphic tangent space $\Theta_{\tau, \mathcal{T}_{L}(M)}$ at each point $\tau \in \mathcal{T}_{L}(M)$ is naturally identified with $H^{1}\left(M_{\tau}, \Theta_{M_{\tau}}\right)$ and $\boldsymbol{C}$. Let $\pi_{\mathcal{C}}: \mathcal{Y} \rightarrow \mathcal{C}$ be any complex analytic family of marked polarized $C Y$ manifolds such that there exists a point $x_{0} \in \mathcal{C}$ and the fibre $\left(\pi_{\mathcal{C}}\right)^{-1}\left(x_{0}\right)=M_{x_{0}}$ as a marked polarized CY manifold is isomorphic to some fibre of the family (7). Then there exists a unique holomorphic map of families

$$
\kappa:(\mathcal{Y} \rightarrow \mathcal{C}) \rightarrow\left(\mathcal{U}_{L} \rightarrow \mathcal{T}_{L}(M)\right)
$$

defined up to a biholomorphic map $\phi$ of the fibres which induces the identity map on $H_{n}(M, \mathbb{Z})$. The restriction of the map $\kappa$ on the base $\mathcal{C}$ is unique.

Proof: In this paragraph we will use the following result of Y.-T. Siu. See [9] and [35].

Theorem 8 Let $X$ be an algebraic variety and let $\mathcal{L}$ be an ample line bundle then $\left(\mathcal{K}_{X}\right)^{\otimes 2} \otimes(\mathcal{L})^{\otimes m}$ is very ample for any

$$
m \geq 2+\left(\begin{array}{c}
3 n+1 \\
n
\end{array}\right)
$$

where $n$ is the dimension of $X$.

Based on Theorem 8 we will prove the following Theorem:

Theorem 9 Let $M$ be a fixed projective manifold with $c_{1}(M)=0$. Let us fix its cohomology ring $H^{*}(M, \mathbb{Z})$ over $\mathbb{Z}$, its Chern classes and the polarization class $L \in H^{2}(M, \mathbb{Z})$ corresponding to an ample line bundle. Then there are only finite number of components of the Hilbert scheme that parametrizes all polarized projective manifolds with fixed cohomology $\operatorname{ring} H^{*}(M, \mathbb{Z})$, and Chern classes with a polarization class $L$.

Proof: Let us fix the following data; a projective manifold $\mathrm{M}$ with canonical class zero, its cohomology ring $H^{*}(\mathrm{M}, \mathbb{Z})$ over $\mathbb{Z}$, its Chern classed and the polarization class $L \in H^{2}(\mathrm{M}, \mathbb{Z})$ which is the Chern class of an ample line bundle. According to a theorem of Sullivan there are only finite number of $C^{\infty}$ structures on $\mathrm{M}$ if the real dimension of $\mathrm{M}$ is greater or equal to 5 with the data mentioned above. See [38]. Suppose that $m$ satisfy the inequality 
(9). Since $\mathcal{L}^{\otimes m}$ is a very ample line, $H^{k}\left(\mathrm{X}, \mathcal{L}^{\otimes m}\right)=0$ for $k>0$ and Theorem 8, imply that if we consider a CY manifold with fixed Chern classes $c_{1}=0$, $c_{2}, \ldots, c_{n}$, fixed cohomology ring $H^{*}(\mathrm{M}, \mathbb{Z})$ and polarization class $L$, then all these Kähler manifolds can be embedded in a fixed projective space $\mathbb{C P}^{k}$. The Hirzebruch-Riemann-Roch theorem implies that for all Kähler manifolds with canonical class zero with fixed cohomology $\operatorname{ring} H^{*}(\mathrm{M}, \mathbb{Z})$, fixed Chern classes and the line bundle $\mathcal{L}$ with a fixed ample Chern class $L$ its the Euler characteristics:

$$
\chi\left(\mathrm{M}, \mathcal{L}^{m}\right)=\operatorname{dim}_{\mathbb{C}} H^{0}\left(\mathrm{M}, \mathcal{L}^{m}\right)=\int_{\mathrm{M}} \operatorname{Td}(\mathrm{M}) C h\left(\mathcal{L}^{m}\right)
$$

have the same Hilbert polynomial $\chi\left(\mathrm{M}, \mathcal{L}^{m}\right)$ for all such manifolds. By using the theory of Hilbert schemes of Grothendieck developed in [12], we can conclude that there are a finite number of components of the Hilbert scheme that parametrizes all polarized Kähler manifolds with fixed cohomology ring $H^{*}(\mathrm{M}, \mathbb{Z})$, Chern classes and the polarization class $L$.

In [17] we proved the following Theorem:

Theorem 10 Let $\mathcal{H}_{L}$ be the Hilbert scheme of non-singular $C Y$ manifolds embedded by the linear system $\left|L^{m}\right|$ defined by the polarization class $L$, then $\mathcal{H}_{L}$ is a non-singular quasi-projective variety.

We know from the results in [12] that there exists a family of polarized CY manifolds:

$$
\mathcal{Y}_{L} \rightarrow \mathcal{H}_{L}
$$

where $\mathcal{Y}_{L} \subset \mathbb{C P}^{N} \times \mathcal{H}_{L}$. Let $\widetilde{\mathcal{H}}_{L}$ be the universal covering of $\mathcal{H}_{L}$ and let

$$
\widetilde{\mathcal{Y}}_{L} \rightarrow \widetilde{\mathcal{H}}_{L}
$$

be the pullback family of (10). It is easy to see that the group $\mathbb{S L}_{N+1}(\mathbb{C})$ acts on $\mathcal{H}_{L}$. This implies that $\mathbb{S L}_{N+1}(\mathbb{C})$ acts also on $\widetilde{\mathcal{H}}_{L}$. We will need the following lemma and its corollary:

Lemma 11 Let $G$ be a subgroup of $\mathbb{S L}_{N+1}(\mathbb{C})$ that fixes a point $\tau_{0} \in \widetilde{\mathcal{H}}_{L}$, then $G$ is a finite group of complex analytic automorphisms of the $C Y$ manifold $M_{\tau_{0}}$ and it is a normal subgroup of $\mathbb{S L}_{N+1}(\mathbb{C})$ that acts trivially on $H_{n}(M, \mathbb{Z})$.

Proof: According to Theorem $10 \mathcal{H}_{L}$ is a smooth quasi-projective variety. So its universal cover $\widetilde{\mathcal{H}}_{L}$ is a simply connected complex manifold and we may suppose that the family (11) is marked and polarized. The 
Definition 5 of the marked family of CY manifolds implies that if $G$ fixes the point $\tau_{0} \in \widetilde{\mathcal{H}}_{L}$, then $G$ is a subgroup in $\mathbb{S L}_{N+1}(\mathbb{C})$ that stabilizes $\mathrm{M}_{\tau_{0}}$ in $\mathbb{C P}^{N}$. This shows that $G$ must be a group of holomorphic automorphisms of $\mathrm{N}_{\tau_{0}}$ and it must act trivially on $H_{n}(\mathrm{M}, \mathbb{Z})$. Theorem 3 implies that $G$ acts trivially on $\widetilde{\mathcal{H}}_{L}$. This will imply that it is a normal subgroup in $\mathbb{S L}_{N+1}(\mathbb{C})$. To show this fact we need to prove that for any element $g \in \mathbb{S L}_{N+1}(\mathbb{C})$ we have $g^{-1} G g=G$. Let $\tau=g^{-1}\left(\tau_{0}\right)$. Then direct computations show that for any element $h \in G$ we have $g^{-1} h g(\tau)=g^{-1} \circ g(\tau)=\tau$. The last equality shows that $G$ is a normal subgroup. Lemma 11 is proved.

Corollary 12 The group $G_{1}:=\mathbb{S L}_{N+1}(\mathbb{C}) / G$ acts freely on $\widetilde{\mathcal{H}}_{L}$.

We will prove that the quotients $\widetilde{\mathcal{Y}}_{L} / G_{1}$ and $\widetilde{\mathcal{H}}_{L} / G_{1}$ exist as complex manifolds and that $\mathcal{U}_{L}:=\widetilde{\mathcal{Y}}_{L} / G_{1} \rightarrow \mathcal{T}_{L}(N):=\widetilde{\mathcal{H}}_{L} / G_{1}$ will be the family (7) with the properties stated in the Theorem.

Palais proved in [28] the following theorem:

Theorem 13 Suppose that an arbitrary Lie group $\mathfrak{G}$ acts on a complex manifold $Y$, then the quotient $Y / \mathfrak{G}$ exists in the category of complex spaces, provided that the map

$$
\psi: \mathfrak{G} \times Y \rightarrow Y \times Y
$$

defined as $\psi(g, m)=(g(m), m)$ is proper.

In order to prove that the morphism defined by the action of the Lie group $\mathfrak{G}=G_{1}:=\mathbb{S L}_{k+1}(\mathbb{C}) / G$ acts properly on $\widetilde{\mathcal{H}}_{L}$ and $\widetilde{\mathcal{Y}}_{L}$, we need to use the following criterion for the properness of the map that can be found in [11], Chapter II, 7:

Criterion 14 Let $\psi: X \rightarrow Z$ be a morphism of algebraic varieties over an algebraically closed field $k, \mathcal{O}$ a discrete valuation ring with a residue class field $k, \phi^{*}: \operatorname{Spec} \mathcal{O} \rightarrow X$ is a rational morphism and $h: \operatorname{Spec} \mathcal{O} \rightarrow Z$ is a morphism, where $\psi \circ \phi^{*}=h$. If for such $\phi^{*}$ and $h$ there exists a morphism $\phi: \quad \operatorname{Spec} \mathcal{O} \rightarrow X$, coinciding with $\phi^{*}$ on the generic point of Spec $\mathcal{O}$, the morphism $f$ is proper.

To apply the Criterion 14 in our situation we notice that the properness of the map (12) for the manifolds $\widetilde{\mathcal{Y}}_{L}$ and $\widetilde{\mathcal{H}_{L}}$ and the group $G_{1}$ is an obvious formal consequence of the analogous fact for the varieties $\mathcal{Y}_{L}$ and $\mathcal{H}_{L}$. In this case we have to deal with algebraic varieties and algebraic action of the group $G_{1}$. Therefore $\psi$ is a morphism in the category of algebraic varieties. The existence of the Grothendieck families $\mathcal{Y}_{L} \rightarrow \mathcal{H}_{L}$ defines the 
following families $\pi_{1}: \mathcal{N}_{L} \rightarrow G_{1} \times \mathcal{H}_{L}$ and $\pi_{2}: \mathcal{R}_{L} \rightarrow \mathcal{H}_{L} \times \mathcal{H}_{L}$, where $\pi_{1}^{-1}(g, \tau):=\mathrm{N}_{g(\tau)} \times \mathrm{N}_{\tau}$ and $\pi_{2}^{-1}\left(\tau_{1}, \tau_{2}\right)=\mathrm{N}_{\tau_{1}} \times \mathrm{N}_{\tau_{2}}$.

Next we are going to apply Criterion 14 to the map $\psi$ defined by (12). We will need to use Theorem 2 of the paper [26] of Matsusaka and Mumford. It asserts that if $V$ and $W$ are smooth polarized varieties over $S p e c \mathcal{O}$, neither of which is ruled, and their specialization $V_{0}$ and $W_{0}$ over the closed point of $\operatorname{Spec} \mathcal{O}$ are also smooth polarized varieties, then the specialization of any isomorphism $\psi: V \rightarrow W$ is an isomorphism $\psi_{0}: V_{0} \rightarrow W_{0}$.

It is easy to see that giving a morphism of algebraic varieties $h$ : $\operatorname{Spec} \mathcal{O} \rightarrow \mathcal{H}_{L} \times \mathcal{H}_{L}$ and a rational morphism $\phi^{*}: \operatorname{Spec} \mathcal{O} \rightarrow G_{1} \times \mathcal{H}_{L}$ satisfying the criterion stated in the above lemma, defines varieties $V$ and $W$ over $\operatorname{Spec} \mathcal{O}$ and the isomorphism $\psi^{*}$ of them. On the other hand $\phi^{*}$ gives a rational morphism of $\operatorname{Spec} \mathcal{O}$ into $G_{1}$ and therefore a rational morphism $\phi^{*}$ of $\operatorname{Spec} \mathcal{O}$ into the projective closure of $\bar{G}_{1}$ of the projective group $G_{1}$. Since the dimension of the scheme $\operatorname{Spec} \mathcal{O}$ is one, then the rational morphism $\phi^{*}$ of $\operatorname{Spec} \mathcal{O}$ into a projective variety $\bar{G}_{1}$ can be prolonged to a morphism $\phi$ of $\operatorname{Spec} \mathcal{O}$ to $\bar{G}_{1}$. Thus we get a family $\bar{W} \rightarrow \operatorname{Spec} \mathcal{O}$ which is defined over the closed point of $\operatorname{Spec} \mathcal{O}$ and contains the family $W \rightarrow \operatorname{Spec} \mathcal{O}$. The fact that the rational morphism $\phi^{*}$ can be prolonged to an algebraic morphism $\phi$ implies that the morphism $\psi^{*}$ can be prolonged to a morphism $\psi$ between the varieties $V$ and $\bar{W}$. By the Theorem of Matsusaka and Mumford, the specialization of $\psi$ is an isomorphism, i.e. $\psi$ takes closed point of $\operatorname{Spec} \mathcal{O}$ into a point of $G_{1}$. This means that $\psi$ is an algebraic morphism of $\operatorname{Spec} \mathcal{O}$ into $G_{1}$. Thus the criterion of properness for the morphism $\psi$ holds. As we pointed out this fact implies that the action of $G_{1}$ on $\widetilde{\mathcal{H}}_{L}$ is proper too The quotient $\widetilde{\mathcal{H}}_{L} / G_{1}$ exists and it is a smooth algebraic variety.

The situation is analogous to the morphism $\pi: \widetilde{\mathcal{Y}}_{L} \rightarrow \mathcal{U}_{L}=\widetilde{\mathcal{Y}}_{L} / G_{1}$. Thus we have proved that the quotients $\widetilde{\mathcal{Y}}_{L} / G_{1}$ and $\widetilde{\mathcal{H}}_{L} / G_{1}$ exist as complex spaces. It is obvious that they define a family $\mathcal{U}_{L} \rightarrow \mathcal{T}_{L}(\mathrm{M})$ of marked polarized CY manifolds. According to Cor. 12, $G_{1}$ acts on $\mathcal{Y}_{L}$ and $\mathcal{H}_{L}$ without fixed points. Therefore,

$$
\widetilde{\mathcal{Y}}_{L} / G_{1}=\mathcal{U}_{L} \text { and } \tilde{\mathcal{H}}_{L} / G_{1}=\mathcal{T}_{L}(\mathrm{M})
$$

are manifolds. Thus we have constructed the family (7) as required in Theorem 10 . We also proved that the base of the family $(7) \mathcal{T}_{L}(\mathrm{M})$ is smooth. Next we will prove that the complex dimension of $\mathcal{T}_{L}(\mathrm{M})$ is $h^{n-1,1}$ and that the tangent space at each point $\tau \in \mathcal{T}_{L}(\mathrm{~N})$ is isomorphic to $H^{1}\left(\mathrm{M}_{\tau}, \Theta_{\mathrm{M}_{\tau}}\right)$.

We shall prove that the family ( 7$)$ is effectively parametrized. According to Kodaira this means that for any point $s \in \mathcal{T}(\mathrm{M})$ the mapping $T_{s, \mathcal{T}_{L}(\mathrm{M})} \rightarrow$ $H^{1}\left(\mathrm{M}_{s}, \Theta_{\mathrm{M}_{s}}\right)$ is an isomorphism. From the construction of $\mathcal{T}_{L}(\mathrm{M})$ as defined 
in (13) we can conclude that $T_{s, \mathcal{T}_{L}(\mathrm{M})} \approx H^{0}\left(\mathrm{M}, \mathcal{N}_{\mathbb{C P}^{N}} / \mathrm{M}\right) / \operatorname{sl}(N+1)$ where $\operatorname{sl}(N+1)$ is the Lie algebra of the group $\mathbb{S L}(N+1)$. In [17] we proved that

$$
H^{0}\left(\mathrm{M}, \mathcal{N}_{\mathbb{C P}^{N} / \mathrm{M}}\right) / \operatorname{sl}(N+1) \approx H^{1}\left(\mathrm{M}, \Theta_{\mathrm{M}}\right)
$$

Theorem 10 is proved.

Corollary 15 Let $\mathcal{Y} \rightarrow X$ be any family of marked $C Y$ manifolds, then there exists a unique holomorphic map $\phi: X \rightarrow \mathcal{T}_{L}(M)$ up to a biholomorphic map $\psi$ of $M$ which induces the identity map on $H_{n}(M, \mathbb{Z})$.

From now on we will denote by $\mathcal{T}(\mathrm{M})$ the irreducible component of the Teichmüller space that contains our fixed CY manifold M.

\subsection{Construction of the Moduli Space of Polarized CY Man- ifolds}

Definition 16 We will define the mapping class group $\Gamma(M)$ of any compact $C^{\infty}$ manifold $M$ as follows: $\Gamma=D i f f_{+}(M) / D i f f_{0}(M)$, where Dif $f_{+}(M)$ is the group of diffeomorphisms of $M$ preserving the orientation of $M$ and Dif $f_{0}(M)$ is the group of diffeomorphisms isotopic to identity.

Definition 17 Let $L \in H^{2}(M, \mathbb{Z})$ be the imaginary part of a Kähler metric. Let $\Gamma_{L}:=\{\phi \in \Gamma(M) \mid \phi(L)=L\}$.

It is a well-known fact that the moduli space of polarized algebraic manifolds $\mathfrak{M}_{L}(\mathrm{M})$ is $\mathcal{T}_{L}(\mathrm{M}) / \Gamma_{L}$.

Theorem 18 There exists a subgroup of finite index $\Gamma$ of $\Gamma_{L}$ such that $\Gamma$ acts freely on $\mathcal{T}(M)$ and $\Gamma \backslash \mathcal{T}_{L}(M)=\mathcal{M}_{L}(M)$ is a non-singular quasiprojective variety.

Proof: There are homomorphisms induced by the action of the diffeomorphism group on the middle homology groups with coefficients in $\mathbb{Z}: \phi: \Gamma_{L} \rightarrow \mathbb{S} p\left(2 b_{n}, \mathbb{Z}\right)$ and $\phi: \Gamma_{L} \rightarrow \mathbb{S O}(2 p, q ; \mathbb{Z})$ for odd and even dimensional complex Kähler manifolds correspondingly. An important theorem due to Sullivan proved in [38] states that:

Theorem 19 Suppose that the real dimension of a $C^{\infty}$ manifold $M$ is bigger or equal to 5 , then the image $\phi\left(\Gamma_{L}\right)$ of the mapping class group is an arithmetic group. 
This result of Sullivan implies that $\Gamma_{L}$ is an arithmetic group. This means that the image of $\Gamma_{L}$ has a finite index in the groups $\mathbb{S} p\left(2 b_{n}, \mathbb{Z}\right)$, $\mathbb{S O}(2 p, q ; \mathbb{Z})$ and $\operatorname{ker}(\phi)$ is a finite group. A theorem of Borel implies that we can always find a subgroup of finite index $\Gamma$ in $\Gamma_{L}$ such that $\Gamma$ acts freely on $\mathbb{S} p\left(2 b_{n}, \mathbb{R}\right) / \mathbb{U}\left(b_{n}\right)$ or on $\mathbb{S O}_{0}(2 p, q ; \mathbb{R}) / \mathbb{S O}(2 p) \times \mathbb{S O}(q)$.

Lemma $20 \Gamma$ acts without fixed point on $\mathcal{T}_{L}(M)$.

Proof: Let $\mathcal{K}$ be the Kuranishi space of the deformations of M. Suppose that there exists an element $g \in \Gamma$, such that $g(\tau)=\tau$ for some $\tau \in$ $\mathcal{K} \subset \mathcal{T}_{L}(\mathrm{M})$. From the local Torelli theorem we deduce that we may assume that the Kuranishi space $\mathcal{K}$ is embedded in $\mathcal{G}$, the classifying space of the Hodge structures of weight $\mathrm{n}$ on $H^{n}(\mathrm{M}, \mathbb{Z}) \otimes \mathbb{C}$. Griffiths proved in [15] that $\mathcal{G} \approx G / K$ where $\mathrm{G}$ in the odd dimensional case is $\mathbb{S} p\left(2 b_{n}, \mathbb{R}\right)$ and in the even dimensional is $\mathbb{S O}_{0}(2 p, q ; \mathbb{R})$ and $K$ is a compact subgroup of $G$. Let $K_{0}$ be the maximal compact subgroup of $G$. So we have a natural $C^{\infty}$ fibration

$$
K_{0} / K \subset G / K \rightarrow G / K_{0}
$$

Griffith's transversality theorem implies that $\mathcal{K}$ is transversal to the fibres $K_{0} / K$ of the fibration $G / K \rightarrow G / K_{0}$. If $g \in \Gamma$ fixes a point $\tau \in G / K_{0}$, then $g \in K_{0} \cap \Gamma$. On the other hand side it is easy to see that the local Torelli theorem implies that the action of $\Gamma$ on $\mathcal{K}$ is induced from the action $\Gamma$ on $G / K$ by left multiplications. So we can conclude that the action of $\Gamma$ preserves the fibration (14). Lemma 20 follows directly from here and the fact that $\Gamma$ acts without fix point on $G / K_{0}$.

The second part of the theorem, namely that the space $\Gamma \backslash \mathcal{T}_{L}(\mathrm{M})$ is a quasi projective follows directly from the fact that $\Gamma \backslash \mathcal{T}_{L}(\mathrm{M}) \rightarrow \Gamma_{L} \backslash \mathcal{T}_{L}(\mathrm{M})$ is a finite map and that $\Gamma_{L} \backslash \mathcal{T}_{L}(\mathrm{M})$ is a quasi projective variety according to [41]. Theorem 18 is proved.

Theorem 21 There exists a finite cover $\mathcal{M}_{L}(M)$ of $\mathfrak{M}_{L}(M)=\mathcal{T}_{L}(M) / \Gamma_{L}$ with the following properties: $\boldsymbol{A} . \mathcal{M}_{L}(M)$ is a smooth algebraic variety, $\boldsymbol{B}$. Over $\mathcal{M}_{L}(M)$ there exists a family

$$
\mathcal{N}_{L} \rightarrow \mathcal{M}_{L}(M)
$$

of polarized CY manifolds with the following property; Let

$$
\pi_{\mathcal{C}}: \mathcal{Y} \rightarrow \mathcal{C}
$$

We suppose that $K$ or $K_{0}$ acts on the right on $G$ and $\Gamma$ acts on the left on $G$. 
be any complex analytic family of polarized CY manifolds with a class of polarization $L$ such that at least one of its fibres of the family (16) is isomorphic as a polarized variety to a fibre of the family (15). Then there exists a unique complex analytic map $\phi: \mathcal{C} \rightarrow \mathcal{M}_{L}(M)$, which induces a holomorphic map between the families (16) and (15). The map between the families is defined uniquely up to a biholomorphic map $\phi$ of $M$ which induces the identity map on $H_{n}(M, \mathbb{Z})$.

Proof: Let $\psi: \Gamma_{L} \rightarrow H^{n}(\mathrm{M}, \mathbb{Z})$ be the natural representation of the group $\Gamma_{L}$. The results of Sullivan imply that the image $\psi\left(\Gamma_{L}\right)$ is an arithmetic group. According to Theorem 18 there exists a subgroup $\Gamma_{L}^{\prime \prime}$ of finite index in $\psi\left(\Gamma_{L}\right)$ such that $\psi\left(\Gamma_{L}\right)$ acts freely on the Teichmüller space $\mathcal{T}_{L}(\mathrm{M})$. From here we deduce that the space $\Gamma_{L}^{\prime \prime} \backslash \mathcal{T}_{L}(\mathrm{M})$ is smooth. Let us define $\Gamma_{L}^{\prime}:=\psi^{-1}\left(\Gamma_{L}^{\prime \prime}\right)$. Clearly $\Gamma_{L}^{\prime}$ is a subgroup of finite index in $\Gamma_{L}$. The local Torelli theorem implies that $\mathcal{T}_{L}(\mathrm{M}) / \Gamma_{L}^{\prime}$ will be a non-singular variety. From the definition of $\psi$, it follows that $\operatorname{ker} \psi=G$ acts trivially on $H^{n}(\mathrm{M}, \mathbb{Z})$. The local Torelli Theorem implies that $G$ acts trivially on the Teichmüller space $\mathcal{T}_{L}(\mathrm{M})$. The existence and the properties of the family $\mathcal{N}_{L} \rightarrow \mathcal{M}_{L}(\mathrm{M})$ follows from the existence and the properties of the family $\mathcal{Z}_{L} \rightarrow \mathcal{T}_{L}(\mathrm{M})$ proved in Theorem 15.

According to [41] the coarse moduli space $\mathfrak{M}_{L}(\mathrm{M})$ is a quasi-projective variety. Since $\mathcal{M}_{L}(\mathrm{M})$ is a finite cover of $\mathfrak{M}_{L}(\mathrm{M})$, then $\mathcal{M}_{L}(\mathrm{M})$ will be quasi-projective too. Hironaka's Theorem about resolution of singularities states that we can always compactify both $\mathcal{M}_{L}(\mathrm{M})$ and $\mathfrak{M}_{L}(\mathrm{M})$ such that

$$
\overline{\mathcal{M}_{L}(\mathrm{M})} \ominus \mathcal{M}_{L}(\mathrm{M})=\mathcal{D}_{\infty} \text { and } \overline{\mathfrak{M}_{L}(\mathrm{M})} \ominus \mathfrak{M}_{L}(\mathrm{M})=\mathfrak{D}_{\infty}
$$

the divisors $\mathcal{D}_{\infty}$ and $\mathfrak{D}_{\infty}$ are divisors with normal crossings.

\section{Moduli of Maps}

\subsection{Smoothness of the Moduli Space of Maps}

Let $\mathrm{C}$ and $\mathrm{X}$ be two projective varieties. Let $f: \mathrm{C} \rightarrow \mathrm{X}$ be a morphism between them. Let $\Gamma_{f} \subset \mathrm{C} \times \mathrm{X}$ be the graph of the map $f: \mathrm{C} \rightarrow \mathrm{X}$. According to the results of Grothendieck, the Hilbert scheme of $\Gamma_{f} \subset \mathrm{C} \times \mathrm{X}$ is a projective scheme. See [11].

Definition 22 We will denote the Hilbert scheme of $\Gamma_{f} \subset C \times X$ by $\mathfrak{M}_{f}(C, X)$ and we will call it the moduli space of the map $f$.

The above mentioned results of Grothendieck implies that $\mathfrak{M}_{f}(\mathrm{C}, \mathrm{X})$ is a projective scheme. 
Definition 23 a. Let $f: C \rightarrow X$ be a morphism of projective varieties with $\operatorname{dim}_{\mathbb{C}} C \leq \operatorname{dim}_{\mathbb{C}} X$. Suppose that the morphism $f: C \rightarrow f(C)$ is finite. We say that $f$ admits a non-trivial one parameter deformations if there is a nonsingular projective curve $T$ and a family of algebraic maps $F: T \times C \rightarrow X$ such that for some $t_{0} \in T$ we have $F_{t_{0}}=f$ and the morphism $F: T \times C \rightarrow F(T \times C)$ is finite too. $\boldsymbol{b}$. We will say that the deformation of $f$ is trivial if $F_{t}=f$ for all $t \in T$. c. Two families of maps $F_{1}: T \times C_{1} \rightarrow X$ and $F_{2}: T \times C_{2} \rightarrow X$ are said to be isomorphic if there is a common finite cover $C$ of $C_{1}$ and $C_{2}$ such that the lifts of $F_{1}$ to $T \times C$ and $F_{2}$ to $T \times C$ are isomorphic, meaning there exists a biholomorphic map id $\times g$ from $T \times C$ to itself such that $F_{1}=F_{2} \circ(i d \times g)$.

From now on we will consider only one parameter deformations of maps.

Definition 24 Let $C$ be a fixed Riemann surface. Let $X$ be a quasi-projective space and $\bar{X}$ some projective compactification of $X$ such that $\mathcal{D}_{\infty}=\bar{X} \ominus X$ is a divisor with normal crossings. Assume that on $X$ there exists a Kähler metric with non-positive holomorphic bisectional curvature, and negative holomorphic sectional curvature bounded away from zero and logarithmic growth near $\mathcal{D}_{\infty}$. We will consider from now on the set $\mathcal{M}_{C}$ of all holomorphic maps $f: C \rightarrow \bar{X}$ such that: a. the map $f: C \rightarrow f(C)$ is finite map and b. $f(C)$ is not contained in $\mathcal{D}_{\infty}$ and the pullback $f^{*}\left(\mathcal{D}_{\infty}\right)=D_{S}$, where $D_{S}$ is a fixed divisor on $C$.

Theorem 25 Suppose that $X$ is a projective manifold such that on a Zariski open set $X^{\prime}:=\bar{X} \ominus \mathcal{D}_{\infty}$ there exists a Kähler metric with a non-positive holomorphic bi-sectional curvature. Let $C$ be a fixed algebraic curve. Let

$$
\mathcal{M}_{C}:\left\{f: C \rightarrow X \mid f \text { is a finite hol map from } C \text { to } f(C) \text { and } f(C) \varsubsetneqq \mathcal{D}_{\infty}\right\} \text {. }
$$

Then the moduli space $\mathcal{M}_{C}$ is a smooth quasi-projective variety.

Proof: The proof is based on several lemmas.

Lemma 26 Let $f: C \rightarrow X$ be a finite map from an algebraic curve $C$ to $f(C)$ such that $f(C) \varsubsetneqq \mathcal{D}_{\infty}$. Let $\sigma \in H^{0}\left(C, f^{*}\left(T_{X}\right)\right)$ be a non-zero section with a bounded norm. Then $\sigma$ is parallel with respect to the connection induced by the restriction of the Kähler metric on $X \ominus \mathcal{D}_{\infty}$.

Proof: Denote by $\mathrm{C}^{0}=\mathrm{C} \ominus \mathrm{f}^{*}\left(\mathfrak{D}_{\infty}\right)$. Let $\sigma \in H^{0}\left(\mathrm{C} \ominus \mathrm{f}^{*}\left(\mathrm{f}_{*}(\mathrm{C}) \cap \mathcal{D}_{\infty}\right), f^{*}\left(T_{\mathrm{X}}\right)\right)$ be a non-zero section. Suppose that $\nabla \sigma \neq 0$, where $\nabla$ is the covariant derivative on the vector bundle $\left.\mathrm{f}^{*}\left(\mathrm{~T}_{\mathrm{X}}\right)\right|_{\mathrm{C}^{0}}$ induced by the pullback of the Kähler metric on X. Direct computations show that the following formula holds: 


$$
\frac{d^{2}}{d \tau \overline{d \tau}}\|\sigma\|^{2}=\|\nabla \sigma\|^{2}-\left\langle R\left(\frac{d}{d \tau}, \frac{\bar{d}}{d \tau}\right) \sigma, \sigma\right\rangle \geq 0
$$

where $\left\langle R\left(\frac{d}{d \tau}, \frac{\bar{d}}{d \tau}\right) \sigma, \sigma\right\rangle$ is the corresponding holomorphic bi-sectional curvature on the vector bundle $\left.\mathrm{f}^{*}\left(\mathrm{~T}_{\mathrm{X}}\right)\right|_{\mathrm{C}^{0}}$ with a metric induced by the pullback of the Kähler metric on X. Formula (19) and the assumption that we can pro-

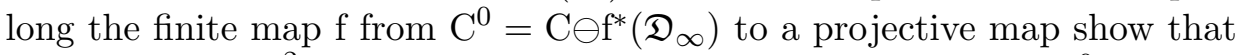
the function $\|\sigma\|^{2}$ is a bounded plurisubharmonic function on $\mathrm{C}^{0}$. Therefore by maximal principle it is the constant function because formula (19) implies

$$
\frac{d^{2}}{d \tau \overline{d \tau}}\|\sigma\|^{2}=0
$$

So we get a contradiction. This implies that $\sigma=0$ if $\sigma$ is not parallel. So if $\sigma$ is a holomorphic non-zero section with finite $L^{2}$ norm, then it must be parallel. Lemma 26 is proved.

Corollary 27 The tangent space at the point $f \in \mathcal{M}_{C}$ is isomorphic to all parallel sections $\sigma \in H^{0}\left(C, f^{*}\left(T_{X}\right)\right)$.

Lemma 28 Let $\sigma \in H^{0}\left(C \ominus f^{*}\left(f(C) \cap \mathcal{D}_{\infty}\right), f^{*}\left(T_{X}\right)\right)$. be a parallel section. Then there exists a family of maps $F_{t}: C \rightarrow X$ where $t \in \mathcal{D}$ and $\mathcal{D}$ is the unit disk such that $F_{0}=f$ and $\left.\frac{d}{d t} F_{t}\right|_{t=0}=\sigma$.

Proof: Since $\sigma \in H^{0}\left(\mathrm{C} \ominus \mathrm{f}^{*}\left(\mathrm{f}(\mathrm{C}) \cap \mathcal{D}_{\infty}\right), \mathrm{f}^{*}\left(T_{\mathrm{X}}\right)\right)$ is a parallel section, there exists a vector $\vec{\tau} \in T_{x, \mathrm{X}}$ at each point $x \in \mathrm{f}(\mathrm{C}) \ominus \mathcal{D}_{\infty}$ such that $\mathrm{f}^{*}(\vec{\tau})=\sigma$ and $x \in \mathrm{f}(\mathrm{C})$. Since the metric $g$ on $\mathrm{X}$ is Kähler we can define as in the Appendix I 7, Prop. 56 the exponential complex map: $\exp _{x}: T_{x, \mathrm{X}} \rightarrow \mathrm{X}$ at each point $x \in \mathrm{f}(\mathrm{C})$ such that $\exp _{x}(t \vec{\tau})=D_{x}$ is a totally geodesic disk when $|t|<1$ and the tangent vector of the disk at the point $x$ is $\vec{\tau}$. The existence of a metric with holomorphic non positive bi-sectional curvature on the moduli space of polarized CY implies that the complex exponential map is injective for $|t|<1$. Since we assumed that $\sigma$ is a parallel section of the vector bundle $\mathrm{f}^{*} T_{\mathrm{X}}$ on $\mathrm{C} \ominus \mathrm{f}^{*}\left(\mathrm{f}(\mathrm{C}) \cap \mathcal{D}_{\infty}\right)$ we deduce immediately that by defining $\mathrm{f}_{t}(\mathrm{~s})=\exp _{\mathrm{f}(\mathrm{s})}(t \vec{\tau})$ and taking the exponential map at each point $x \in f(\mathrm{C})$, we have constructed a one parameter deformation $\mathrm{f}_{t}: \mathrm{C} \times \mathcal{D} \rightarrow \mathrm{X}$ of $\mathrm{f}: \mathrm{C} \rightarrow \mathrm{X}$ as required in lemma 28 .

Lemma 28 and Corollary 27 imply that the moduli space of all the maps $\mathrm{f}: \mathrm{C} \rightarrow \mathrm{X}$ such that $\mathrm{f}(\mathrm{C}) \subsetneq \mathcal{D}_{\infty}$ is smooth. Indeed we know that the tangent space $T_{\mathrm{f}, \mathcal{M}_{\mathrm{C}}}$ of the moduli space $\mathcal{M}_{\mathrm{C}}$ at $\mathrm{f} \in \mathcal{M}_{\mathrm{C}}$ is isomorphic to $H^{0}\left(\mathrm{C}, \mathrm{f}^{*}\left(T_{\mathrm{X}}\right)\right)$. Cor. 27 tells us that $T_{\mathrm{f}, \mathcal{M}_{\mathrm{C}}}$ consists of all parallel sections. 
From Lemma 28 it follows that each parallel section can be integrated to a family of maps parametrized by the unit disk. Grothendieck's theory of Hilbert schemes tells us that the Hilbert scheme of all maps $\mathrm{f}: \mathrm{C} \rightarrow \mathrm{X}$ such that $\mathrm{f}(\mathrm{C}) \nsubseteq \mathcal{D}_{\infty}$ is a quasi-projective space. Theorem 25 is proved.

\subsection{Some Applications}

Let $\left(\mathrm{C} ; x_{1}, . ., x_{n}\right)$ be a Riemann surface with $\mathrm{n}$ distinct fixed points on it. Let $\mathcal{X}_{\mathrm{C}} \rightarrow \mathrm{C}$ be a family of polarized CY manifolds with degenerate fibres over the points $x_{1}, . ., x_{n}$. It is easy to see that the family $\mathcal{X} \rightarrow \mathrm{C}$ defines a map

$$
f: \mathrm{C} \ominus\left(x_{1} \cup \ldots \cup x_{n}\right) \rightarrow \mathcal{T}_{L}(\mathrm{M}) / \Gamma_{L} .
$$

Lemma 29 The map $f$ can be prolonged to a map $\bar{f}: C \rightarrow \overline{\Gamma_{L} \backslash \mathcal{T}_{L}(M)}=$ $\overline{\mathfrak{M}_{L}(M)}$, where the compactification $\overline{\mathfrak{M}_{L}(M)}$ of $\mathfrak{M}_{L}(M)$ is such that $\overline{\mathfrak{M}_{L}(M)}$ is a projective variety and $\overline{\mathfrak{M}_{L}(M)} \ominus \mathfrak{M}_{L}(M)$ is a divisor with normal crossings. [15].

Proof: The proof of Lemma 29 is exactly Proposition 9.10 proved in

Let $\Gamma_{L}$ and $\Gamma$ be the arithmetic groups defined in Theorem 18. The map

$$
\pi: \mathcal{T}_{L}(\mathrm{M}) / \Gamma=\mathfrak{M}_{L}(\mathrm{M}) \rightarrow \mathcal{T}_{L}(\mathrm{M}) / \Gamma_{L}=\mathcal{M}_{L}(\mathrm{M})
$$

is a finite morphism since $\Gamma_{L} / \Gamma$ is a finite set and its cardinality is $N$. We will prove the following lemma:

Lemma 30 There exists a finite affine cover $\psi: C_{1} \rightarrow C \ominus\left(x_{1} \cup \ldots \cup x_{n}\right)$ of degree $N=\# \Gamma_{L} / \Gamma$ such that the map $f$ defined by (21) can be lifted to a map $f_{1}: C_{1} \rightarrow \mathcal{T}_{L}(M) / \Gamma$.

Proof: The construction of the affine Riemann surface $\mathrm{C}_{1}$ is done in a standard way, namely

$$
\mathrm{C}_{1}=\left(\mathrm{C} \ominus\left(x_{1} \cup \ldots \cup x_{n}\right)\right) \times_{f\left(\mathrm{C} \ominus\left(x_{1} \cup \ldots \cup x_{n}\right)\right)} \pi^{-1}\left(f\left(\mathrm{C} \ominus\left(x_{1} \cup \ldots \cup x_{n}\right)\right) .\right.
$$

We know from the local Torelli theorem and the fact $\Gamma_{L} \backslash \mathcal{T}_{L}(\mathrm{M})$ is the moduli space of polarized CY manifolds, that the map $f$ defined by (21) exists. We know that, since $\Gamma \subset \Gamma_{L}$ is a subgroup of finite index, the map $\pi: \Gamma_{L} \backslash \mathcal{T}_{L}(\mathrm{M}) \rightarrow \Gamma \backslash \mathcal{T}_{L}(\mathrm{M})$ is a finite map. Therefore the map $f$ can be lifted to a map $f^{\prime}: \mathrm{C}_{1} \rightarrow \mathcal{T}_{L}(\mathrm{M}) / \Gamma_{L}(\mathrm{M})$. This follows directly from the construction of the open Riemann surface $\mathrm{C}^{\prime}$. Lemma 30 is proved. 
Lemma 31 The map $f_{1}$ constructed in Lemma 30 can be prolonged to a map $f: \overline{C_{1}} \rightarrow \overline{\Gamma \backslash \mathcal{T}_{L}(M)}=\overline{\mathcal{M}_{L}(M)}$ where $\overline{C_{1}}$ is the closure of $C_{1}$ in the compactification $\overline{\mathcal{M}_{L}(M)}$ of $\mathcal{M}_{L}(M)$ as defined in (17).

Proof: The proof of Lemma 31 is the same as Lemma 29 and is due to Griffiths. See [15].

Definition 32 We will say that a family of polarized $C Y$ manifolds $\pi$ : $\mathcal{X}_{C} \rightarrow C$ over a Riemann surface $C$ is rigid if the moduli space of the map $f: C \rightarrow \mathfrak{M}_{L}(M)$ induced by the natural holomorphic map of the base of the family into the moduli space of polarized CY manifolds is a discrete set.

The proof of the following remark is obvious and we will omit it.

Let $\mathcal{X} \rightarrow \mathrm{C}$ be a family of polarized $\mathrm{CY}$ manifolds over a fixed Riemann surface $\mathrm{C}$ with a fixed points of degenerations. Let $\pi_{1}: \mathrm{C}_{1} \rightarrow \mathrm{C}$ be the finite cover constructed in Lemmas 30 and 31 . Let $\mathcal{X}_{1} \rightarrow \mathrm{C}_{1}$ is the pullback of the family $\mathcal{X} \rightarrow \mathrm{C}$. Then the family $\mathcal{X} \rightarrow \mathrm{C}$ is rigid if and only if the pullback family $\mathcal{X}_{1} \rightarrow \mathrm{C}_{1}$ is rigid.

\section{Yukawa Coupling and Rigidity}

Definition 33 Suppose that $\omega_{\tau}$ is a family of holomorphic forms on the local universal family of $C Y$ manifolds over the Kuranishi space of polarized CY manifold. The expression

$$
\langle\underbrace{\left(\nabla_{\frac{\partial}{\partial \tau^{i} 1}} \circ\left(\nabla_{\frac{\partial}{\partial \tau^{i} 2}} \circ \ldots \circ\left(\nabla_{\frac{\partial}{\partial \tau^{i} n}}\left(\omega_{\tau}\right)\right)\right)\right)}_{n}, \omega_{\tau}\rangle
$$

is a holomorphic section of $\left(\left(\Omega_{\mathcal{X} / \mathcal{M}(M)}^{n}\right)^{*}\right)^{\otimes 2} \otimes S^{\otimes n}\left(\mathcal{T}_{\mathcal{M}(M)}\right.$. This section is called the Yukawa coupling.

Condition 34 Suppose that for every $\phi \in H^{1}\left(M, \Theta_{M}\right)$ different from zero the following condition holds:

$$
\wedge^{n} \phi \neq 0 \in H^{n}\left(M, \wedge^{n} \Theta_{M}\right)
$$

Then we will prove that any family $\pi: \mathcal{X}_{C} \rightarrow C$ over a Riemann surface $C$ is rigid if one of its fibre is $M$. 
The condition 34 is difficult to check. Now we will formulate an equivalent condition which is much easier to verify. Let $\pi: \mathcal{X} \rightarrow \mathcal{D}$ be a non-trivial family of non singular $\mathrm{n}$ complex dimensional CY manifolds over the unit disk $\mathcal{D}$. We will denote by $t$ the local coordinate in $\mathcal{D}$. Let us denote by $\nabla_{\frac{\partial}{\partial t}}$ the covariant differentiation induced by the Gauss-Manin connection. According to Kodaira-Spencer-Kuranishi theory the tangent vectors at $0 \in \mathcal{D}$ can be identified with non-zero elements $\phi \in H^{1}\left(\mathrm{M}_{0}, \Theta_{\mathrm{M}_{0}}\right)$. Let $\Omega_{\mathcal{X} / \mathcal{D}}^{n}$ be the relative dualizing sheaf. Let $\omega_{t} \in H^{0}\left(\mathcal{X}, \Omega_{\mathcal{X} / \mathcal{D}}^{n}\right)$ be a family of holomorphic $n$ forms $\omega_{t}$. We may suppose that $\omega_{0} \neq 0$.

Theorem 35 Let $\phi \in H^{1}\left(M, \Theta_{M}\right)$, then the following condition

$$
\langle\left.\underbrace{\left(\nabla_{\frac{\partial}{\partial t}} \circ\left(\nabla_{\frac{\partial}{\partial t}} \circ \ldots \circ\left(\nabla_{\frac{\partial}{\partial t}}\left(\omega_{t}\right)\right)\right)\right)}_{n}\right|_{t=0}, \omega_{0}\rangle \neq 0,
$$

is equivalent to (22), i.e. $\wedge^{n} \phi \neq 0$ in $H^{n}\left(M, \wedge^{n} \Theta_{M}\right)$.

Proof: It is a standard fact that for any family of forms $\omega_{s}$ the covariant differentiation given by the Gauss-Manin connection is given by the formula:

$$
\left.\left.\nabla_{\frac{\partial}{\partial t}}\left(\omega_{t}\right)\right|_{t=0}=\omega_{0}\right\lrcorner \phi
$$

where $\lrcorner$ means contraction of tensors. From (24) we derive directly:

$$
\left.\underbrace{\left(\nabla_{\frac{\partial}{\partial t}} \circ\left(\nabla_{\frac{\partial}{\partial t}} \circ \ldots \circ\left(\nabla_{\frac{\partial}{\partial t}}\left(\omega_{t}\right)\right)\right)\right)}_{n}\right|_{t=0}=\left(\wedge^{n} \phi\right)\lrcorner \omega_{0} .
$$

(25) means that the form $\left.\left(\wedge^{n} \phi\right)\right\lrcorner \omega_{0}$ is of type $(0, n)$. This implies directly that (23) and (22) are equivalent. Theorem 35 is proved.

Theorem 36 Let $\pi: \mathcal{X}_{C} \rightarrow C$ be a non-isotrivial family of $C Y$ manifolds over a compact Riemann surface $C$. Suppose that there exists a point $\tau_{0} \in C$ such that the fibre $\pi^{-1}\left(\tau_{0}\right)=M_{\tau_{0}}$ satisfies Condition 34 . Suppose that $\mathcal{X}_{C}$ is a projective manifold. Then the family $\pi: \mathcal{X}_{C} \rightarrow C$ is rigid.

Proof: Theorem 36 follows from the fact that the moduli space of the deformations of the map $f$ defined in Definition 32 is smooth by Theorem 25 and Lemma 37. The rigidity therefore implies that the tangent space of the moduli space at the point $\mathrm{f}: \mathrm{C} \rightarrow \overline{\mathcal{M}_{L}(\mathrm{M})}$ is zero dimensional.

Lemma 37 Suppose that the family $\pi: \mathcal{X}_{C} \rightarrow C$ satisfies the conditions of Theorem 36, then the set of the parallel sections of the bundle $f^{*}\left(T_{\mathcal{M}_{L}(M)}\right)$ consists only of the zero section. 
Proof: The proof of Lemma 37 is done by contradiction. According to [30] the non-rigidity of the family $\pi: \mathcal{X}_{\mathrm{C}} \rightarrow \mathrm{C}$ means the existence of $\phi \in H^{0}\left(\mathrm{C} \ominus \mathrm{S}, f^{*}\left(T_{\mathcal{M}(\mathrm{M})}\right)\right), \phi \neq 0$ such that $\phi$ is parallel section with respect to the pullback of the Hodge metric on $\mathcal{M}(\mathrm{M})$. Since the tangent space at a point $\tau \in \mathcal{M}(\mathrm{M})$ can be identified with $H^{1}\left(\mathrm{M}_{\tau}, T_{\mathrm{M}}^{1,0}\right)$, the restriction of the section $\phi$ at each point $\tau \in \mathrm{C} \ominus \mathrm{S}$ can be viewed as a Kodaira-Spencer class $\phi_{\tau} \in H^{1}\left(\mathrm{M}_{\tau}, T_{\mathrm{M}}^{1,0}\right)$. Locally on $\mathrm{M}_{\tau}, \phi_{\tau}$ is given by

$$
\phi_{\tau}:=\sum_{i, j}\left(\phi_{\tau}\right) \frac{i}{j} \overline{d z^{j}} \otimes \frac{d}{d z^{i}} .
$$

On the other hand $\wedge^{n} \phi_{\tau}$ is represented on $\mathrm{M}_{\tau}$ by

$$
\wedge^{n} \phi_{\tau}=\operatorname{det}\left(\left(\phi_{\tau}\right) \frac{i}{j}\right) \overline{d z^{1}} \wedge \ldots \wedge \overline{d z^{n}} \otimes \frac{d}{d z^{1}} \wedge \ldots \wedge \frac{d}{d z^{n}} .
$$

Since $\phi_{\tau} \in C^{\infty}\left(\mathrm{M}, \operatorname{Hom}\left(\Omega_{\mathrm{M}_{\tau}}^{1,0}, \Omega_{\mathrm{M}_{\tau}}^{0,1}\right)\right)$ then

$$
\begin{gathered}
\wedge^{n} \phi_{\tau} \in C^{\infty}\left(\mathrm{M}, \text { Hom }\left(\wedge^{n}\left(\Omega_{\mathrm{M}_{\tau}}^{1,0}\right), \wedge^{n}\left(\Omega_{\mathrm{M}_{\tau}}^{0,1}\right)\right)\right) \approx \\
C^{\infty}\left(\mathrm{M}, \operatorname{Hom}\left(\Omega_{\mathrm{M}_{\tau}}^{n, 0}, \Omega_{\mathrm{M}_{\tau}}^{0, n}\right)\right) \approx C^{\infty}\left(\mathrm{M}_{\tau},\left(\left(\Omega_{\mathrm{M}_{\tau}}^{n, 0}\right)^{*}\right)^{\otimes 2}\right) .
\end{gathered}
$$

Combining (26) and (27) we get that

$$
\wedge^{n} \phi_{\tau} \in H^{0}\left(\mathrm{C},\left(\left(R^{0} \pi_{*}\left(\Omega_{\mathcal{X} / \mathcal{M}(\mathrm{M})}^{n}\right)\right)^{*}\right)^{\otimes 2}\right)
$$

is a non zero class. Moreover according to Lemma $26 \phi_{\tau}$ is a parallel section with respect to the pullback of the Hodge metric. This implies that $\wedge^{n} \phi_{\tau}$ is a global parallel non zero section on $\mathrm{C}$ of the line bundle $\left(\left(R^{0} \pi_{*}\left(\Omega_{\mathcal{X} / \mathcal{M}(\mathrm{M})}^{n}\right)\right)^{*}\right)^{\otimes 2}$ which means that it is a trivial line bundle on $\mathrm{C}$. This is impossible since we know from [25] and the Grothendieck Riemann relative Riemann Roch Theorem that the Chern class $c_{1}\left(\left(R^{0} \pi_{*}\left(\Omega_{\mathcal{X} / \mathcal{M}(\mathrm{M})}^{n}\right)\right)^{*}\right)$ of the line bundle $\left(R^{0} \pi_{*}\left(\Omega_{\mathcal{X} / \mathcal{M}(\mathrm{M})}^{n}\right)\right)^{*}$ is proportional to the imaginary part of the Weil-Petersson metric. We also know that the Chern form of the $L^{2}$ metric on $\left(R^{0} \pi_{*}\left(\Omega_{\mathcal{X} / \mathcal{M}(\mathrm{M})}^{n}\right)\right)^{*}$ is the imaginary part of the WeilPetersson metric. So

$$
\int_{\mathrm{C}} c_{1}\left(\left(R^{0} \pi_{*}\left(\Omega_{\mathcal{X} / \mathcal{M}(\mathrm{M})}^{n}\right)\right)^{*}\right)>0 .
$$


The inequality (29) implies that $R^{0} \pi_{*}\left(\Omega_{\mathcal{X} / \mathcal{M}(\mathrm{M})}^{n}\right)$ is not a trivial line bundle on C. So we get a contradiction. Lemma 37 is proved.

Lemma 37 implies Theorem 36.

Remark. Theorem 36 presumably also follows from the methods used in [43], and it seems to extend to non-isotrivial morphisms $\pi: \mathcal{X}_{\mathrm{C}} \rightarrow \mathrm{C}$ whose generic fibre has a semi-ample canonical sheaf.

Theorem 38 Suppose that $\pi: \mathcal{X} \rightarrow \mathcal{C}$ is a family of polarized $C Y$ manifold over a Riemann surfaces $\mathcal{C}$. Suppose that the family contains a point $\tau_{0} \in \mathcal{C}$ such that around it the monodromy operator $\mathrm{T}$ has a maximal index of unipotency, i.e.

$$
\left(\mathcal{T}^{N}-i d\right)^{n+1}=0 \text { and }\left(\mathcal{T}^{N}-i d\right)^{n} \neq 0
$$

Then the family $\pi: \mathcal{X} \rightarrow \mathcal{C}$ is rigid.

Proof: We will prove that the Yukawa coupling is non-zero in an open neighborhood of the point $\tau_{0} \in \mathcal{C}$ around which the condition (30) holds. Theorem 38 follows from the existence of limit mixed Hodge structure related to the monodromy operator $\mathcal{T}$ established in [36]. Indeed the condition (30) combined with Schmid's Theorem implies the existence of the filtration

$$
\mathbb{W}_{0} \subset \mathbb{W}_{1} \subset \ldots \subset \mathbb{W}_{2 n-1} \subset \mathbb{W}_{2 n}=\mathbb{V} \otimes \mathbb{C}=H^{n}\left(\mathrm{M}_{\tau}, \mathbb{C}\right)
$$

such that the nilpotent operator $\mathcal{N}=\log \left(\mathcal{T}^{N}-i d\right)$ acts on the filtration (31) as follows: $\mathcal{N}\left(\mathbb{W}_{k}\right) \subseteq \mathbb{W}_{k-2}$. In case of CY manifolds Schmid Theorem about the existence of the limit mixed Hodge structure and condition (30) imply that

$$
\mathcal{N}^{n}\left(\mathbb{W}_{2 n} / \mathbb{W}_{2 n-1}\right) \approx \mathbb{W}_{0}
$$

(See [36].) The nilpotent orbit theorem proved in [36] combined with (32) implies that for each point in a "small disk" containing the point $\tau_{0} \in \mathcal{C}$ we have that

$$
\underbrace{\nabla_{\frac{\partial}{\partial \tau_{j_{1}}}} \circ \ldots \circ \nabla_{\frac{\partial}{\partial \tau_{j_{n}}}}}_{n} \omega_{\tau} \neq 0
$$

and the $(0, n)$ component of $(33)$ is a non-zero class of cohomology. Here $\omega_{\tau}$ is a family of holomorphic $n$ forms around the point $\tau \in \mathcal{K}$ in the Kuranishi space that corresponds to the fibre of the point on $\mathcal{C}$ close to $\tau_{0}$. Theorem 38 follows directly from the definition 33 and Theorem 36 . 


\section{Yau's Form of Schwartz Lemma and Bounded- ness}

S.-T. Yau proved in [47] the following theorem, which is a generalization of the Schwarz Lemma:

Theorem 39 Let $N$ be a complex manifold and let $h$ be a Hermitian metric on $N$ such that the holomorphic sectional curvature $K$ of $h$ has the following property; there exists a positive constant $c>0$ such that $K \leq-c$. Let $C$ be either a compact or an affine Riemann surface of hyperbolic type. Let $\phi: C \rightarrow N$ be a holomorphic map different from the constant one, then we have $\phi^{*}(h) \leq c^{-1} g_{P}$, where $g_{P}$ is the Poincare metric on $C$, i.e. the metric with a constant curvature.

This lemma was used in [23] to derive various height inequalities. Here we will use the same method to prove the following Theorem:

Theorem 40 Let $\phi: C \rightarrow \overline{\mathcal{M}_{L}(M)}$ be a holomorphic map such that $\phi(C) \varsubsetneqq$ $\mathcal{D}_{\infty}$ and let $x_{1}, \ldots x_{n}$ be the only $n$ different points on $C$ such that $\phi\left(x_{i}\right) \in$ $\mathcal{D}_{\infty}$. Let $C_{1}:=C \Theta\left(x_{1} \cup \ldots \cup x_{n}\right)$. Then we have the following estimate $\operatorname{vol}\left(\phi\left(C_{1}\right)\right) \leq c^{-1}\left|\chi\left(C_{1}\right)\right|$ for the volume of $\phi\left(C_{1}\right)$ in $\mathcal{M}(M)$ with respect to the Hodge metric $h$ on $\mathcal{M}(M)$.

Proof: From Yau's form of the Schwarz Lemma we obtain:

$$
\operatorname{vol}\left(\phi\left(\mathrm{C}_{1}\right)\right)=\int_{C_{1}} h \leq c^{-1} \int_{C_{1}} g_{p} .
$$

Since the Poincare metric has a constant curvature -1 and from the GaussBonnet theorem we obtain

$$
\int_{C_{1}} g_{p}=\left|\chi\left(\mathrm{C}_{1}\right)\right| .
$$

From this Theorem 40 follows directly.

Definition 41 Let $\left(C ; x_{1}, \ldots, x_{n}\right)$ be a given Riemann surface with fixed points $x_{1}, \ldots, x_{n}$. Let $S$ be the divisor $x_{1}+\ldots+x_{n}$ on $C$. Let us fix a $C Y$ manifold $M$. We will define $S h(C ; S, M)$ to be the set of all possible families $\mathcal{X} \rightarrow C$ of $C Y$ manifolds defined up to an isomorphism over $C$ with fixed degenerate fibres over the points $x_{1}, \ldots, x_{n}$, where $\mathcal{X}$ is a projective manifold and the generic fibre is a $C Y$ manifold $C^{\infty}$ equivalent to $M$. 
Theorem 42 Suppose that Condition 34 or its equivalent (23) holds for some fibre $M_{\tau}$ of a family of CY manifolds over a fix Riemann surface $C$ with fixed points of degenerations Then the set $S h(C ; S)$ is finite.

Proof: The proof of Theorem 42 will be done in two steps. The first step will be to prove that the set of maps $S h(\mathrm{C} ; \mathrm{S})$ is discrete. The second step is to show that for each $\phi \in S h(\mathrm{C} ; \mathrm{S})$ the volume of $\phi(\mathrm{C})$ is bounded by a universal constant. Then Theorem 42 will follow directly from the theorem of Bishop proved in [8], which implies that the set $S h(\mathrm{C}, \mathrm{S})$ is compact.

Lemma 43 The set of maps $S h(C, S)$ is discrete.

Proof: Since we assumed that the CY manifold M satisfies Condition (22), Lemma 43 follows directly from Theorem 36.

We will need the following Lemma for establishing the second step described above:

Lemma 44 Let $S h(C, S)$ be the set defined in Definition 24, where $S$ is the divisor $x_{1}+\ldots+x_{n}$ on $C$. Then $\operatorname{Sh}(C, S)$ is compact.

Proof: Each point $z \in S h(\mathrm{C}, \mathrm{S})$ is represented by a map $\phi_{z}: \mathrm{C} \rightarrow$ $\mathcal{T}(\mathrm{M}) / \Gamma$ which satisfies the conditions of Definition 24. Let $g$ be the Kähler $(1,1)$ form of the Hodge metric on $\mathcal{T}(\mathrm{M}) / \Gamma$, then we can define a height function $h$ on $S h(\mathrm{C}, \mathrm{S})$ as follows:

$$
h\left(\phi_{z}\right)=\int_{\phi\left(\mathrm{C}_{z}\right)} \operatorname{Im} g=\operatorname{vol}\left(\phi_{z}(\mathrm{C})\right) .
$$

Since the metric $g$ is a Kähler metric with logarithmic growth on $\mathrm{X}$, then

$$
h\left(\phi_{z}\right)=\int_{\frac{\mathcal{T}(\mathrm{M}) / \Gamma}{}} \operatorname{Im}(g) \wedge \mathcal{P}(\phi(\mathrm{C}))=\operatorname{vol}\left(\phi_{z}(\mathrm{C})\right) .
$$

where $\mathcal{P}\left(\phi_{z}(\mathrm{C})\right)$ is the Poincare dual of the homology class of $\overline{\phi_{z}(\mathrm{C})}$ in

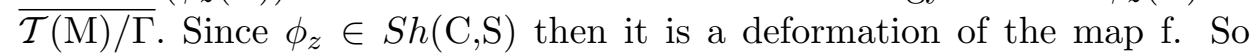
for all $\phi_{z} \in S h(\mathrm{C}, \mathrm{S}), \overline{\phi_{z}(\mathrm{C})}$ realizes a fixed class of homology in $\overline{\mathcal{T}(\mathrm{M}) / \Gamma}$. The integrals that appeared in (34) and (34) are finite. This follows from Theorem 5.1. proved in [25]. Theorem 40 implies that $h\left(\phi_{z}\right)$ is a bounded function on $\operatorname{Sh}(\mathrm{C}, \mathrm{S})$.

We will prove now that $S h(\mathrm{C}, \mathrm{S})$ is a compact set. Since $S h(\mathrm{C}, \mathrm{S})$ is a discrete set, the compactness of $S h(\mathrm{C}, \mathrm{S})$ will imply that it is a finite set. 
The compactness of $S h(\mathrm{C}, \mathrm{S})$ will follows if we prove that from any sequence $\left\{\phi_{n}\right\}$ in $S h(\mathrm{C}, \mathrm{S})$ there exists a subsequence which converges weakly to an algebraic map, i.e. the corresponding subsequence of images of $\mathrm{S}$ converges to an algebraic subvariety of X. Bishop's theorem implies that. See page 292 of [8] and also the Appendix II. Lemma 44 is proved.

Theorem 42 follows directly from Lemmas 43 and 44.

\section{Higgs Bundles, VHS and Rigidity}

We are going to study the relationship between Higgs bundles, Variations of Hodge Structures (VHS) and the existence of non rigid families of CY manifolds. Let $\mathrm{S}^{0}$ and $\mathrm{T}^{0}$ be smooth quasi-projective manifolds. Let

$$
\pi: \mathcal{X} \rightarrow \mathrm{S}^{0} \times \mathrm{T}^{0}=\mathcal{Y}_{0}
$$

be a non rigid family of Calabi-Yau 3-folds, such that the induced map into the moduli space is generically finite. Let $\mathbb{V}=R^{3} \pi_{*} \mathbb{Z}$ denote the flat bundle associated with the the third cohomology group. The Variation of Hodge Structures (VHS) of weight 3 of $\pi$ associated with the family (36) will be $\mathbb{V} \otimes \mathbb{C}$ The corresponding Hodge bundles are then

$$
\mathbb{V} \otimes \mathbb{C}=\pi_{*} \Omega_{\mathcal{X} / \mathcal{Y}_{0}}^{3} \oplus R^{1} \pi_{*} \Omega_{\mathcal{X} / \mathcal{Y}_{0}}^{2} \oplus R^{2} \pi_{*} \Omega_{\mathcal{X} / \mathcal{Y}_{0}}^{1} \oplus R^{3} \pi_{*} \mathcal{O}_{\mathcal{X}}
$$

The cup product of the Kodaira-Spencer class of $\pi \theta_{0}^{p, q}: E_{0}^{p, q} \rightarrow E_{0}^{p, q} \otimes \Omega_{\mathcal{Y}_{0}}^{1}$, where

$E_{0}^{3,0}=\pi_{*} \Omega_{\mathcal{X} / \mathcal{Y}_{0}}^{3}, E_{0}^{2,1}=R^{1} \pi_{*} \Omega_{\mathcal{X} / \mathcal{Y}_{0}}^{2}, E_{0}^{1,2}=R^{2} \pi_{*} \Omega_{\mathcal{X} / \mathcal{Y}_{0}}^{2}$ and $E_{0}^{0,3}=R^{3} \pi_{*} \mathcal{O}_{\mathcal{X}}$

will provide $\left(\underset{p+q=3}{\oplus} E_{0}^{p, q}, \underset{p+q=3}{\oplus} \theta_{0}^{p, q}\right)$ with the Higgs bundle structure on $\mathbb{V}$ corresponding to the VHS.

Theorem 45 Suppose that $S^{0}$ and $T^{0}$ are Zariski open sets in projective varieties $S$ and T. Suppose that

$$
\pi: \mathcal{X} \rightarrow \mathcal{Y}_{0}=S^{0} \times T^{0}
$$

is a non-rigid family of Calabi-Yau manifolds, such that the induced map to the moduli space is generically finite. Let $\mathbb{V} \rightarrow \mathcal{Y}_{0}=S^{0} \times T^{0}$ be the VHS induced by the family (37). Suppose that $\left(s_{i}, t_{j}\right)$ is a point of $\mathfrak{D}_{\infty}=$ $\left(S \ominus S^{0}\right) \times\left(T \ominus T^{0}\right)$. Let $\gamma_{s_{i}}$ and $\gamma_{t_{j}}$ be two short loops around the divisors $T_{s_{i}}$ and $S_{t_{j}}$. Let $S_{t_{0}}$ be the curve in $S$-direction passing through the point $\left(s_{0}, t_{0}\right)$. Suppose that the monodromy operators $\rho\left(\gamma_{s_{i}}\right)=\mathcal{T}_{i}$ and $\rho\left(\gamma_{t_{j}}\right)=\mathcal{T}_{j}$ have infinite order, then the endomorphisms $\mathcal{T}_{i}:\left.\left.\mathbb{V}\right|_{\left(s_{0}, t_{0}\right)} \rightarrow \mathbb{V}\right|_{\left(s_{0}, t_{0}\right)}$ and $\mathcal{T}_{j}:\left.\left.\mathbb{V}\right|_{\left(s_{0}, t_{0}\right)} \rightarrow \mathbb{V}\right|_{\left(s_{0}, t_{0}\right)}$ are distinct. 
Proof: The idea of the proof is to construct on the restrictions of VHS $\left.\mathbb{V}\right|_{\mathrm{S}^{0}}$ and $\left.\mathbb{V}\right|_{\mathrm{T}^{0}}$ two endomorphisms by using the fact that the family of CY manifold $\mathcal{X} \rightarrow \mathrm{S}^{0} \times \mathrm{T}^{0}$ is not rigid. According to Lemma 26 these two endomorphisms are parallel sections of the Hodge bundle $R^{1} \pi_{*} \Omega_{\mathcal{X} / \mathrm{S}^{0} \times \mathrm{T}^{0}}^{n-1}$. Theorem 45 will follow from the way the weight filtrations of nilpotent endomorphisms introduced by W. Schmid are constructed, the fact that the two filtrations will be different and the theorem about the complete reducibility of local systems of VHS over quasi-projective manifolds due to Deligne.

There are two ways to obtain endomorphisms on $\left.\mathbb{V}\right|_{S_{t}}$ and $\left.\mathbb{V}\right|_{\mathrm{T}_{j}}$, where $\mathrm{S}_{i}:=\mathrm{S} \times t_{0}$ and $\mathrm{T}_{j}=s_{0} \times \mathrm{T}$.

1. Consider the two projections $p_{\mathrm{S}}: \mathrm{S} \times \mathrm{T} \rightarrow \mathrm{S}$ and $p_{\mathrm{T}}: \mathrm{S} \times \mathrm{T} \rightarrow \mathrm{T}$. One has

$$
\Omega_{\mathrm{S} \times \mathrm{T}}^{1}\left(\log \mathcal{D}_{\mathrm{S} \times \mathrm{T}}\right)=p_{\mathrm{S}}^{*}\left(\Omega_{\mathrm{S}}^{1}\left(\log \mathcal{D}_{\mathrm{S}}\right)\right) \oplus p_{\mathrm{T}}^{*}\left(\Omega_{\mathrm{T}}^{1}\left(\log \mathcal{D}_{\mathrm{T}}\right)\right)
$$

where $\mathcal{D}_{\mathrm{S} \times \mathrm{T}}$ is the discriminant locus of the family (37). Then the restriction of the Higgs map to $\mathrm{S}_{t}$ defines a natural map

$$
\left.\theta\right|_{\mathrm{S}_{t}}:\left(\left.p_{\mathrm{S}}^{*}\left(\Theta_{\mathrm{S}}\left(-\log \mathcal{D}_{\mathrm{S}}\right)\right) \oplus p_{\mathrm{T}}^{*}\left(\Theta_{\mathrm{T}}\left(-\log \mathcal{D}_{\mathrm{T}}\right)\right)_{\mathrm{S}_{t}} \rightarrow \operatorname{End}(\mathbb{V})\right|_{\mathrm{S}_{t}}\right.
$$

by identifying the sections of $\left(p_{\mathrm{S}}^{*}\left(\Theta_{\mathrm{S}}\left(-\log \mathcal{D}_{\mathrm{S}}\right)\right) \oplus p_{\mathrm{T}}^{*}\left(\Theta_{\mathrm{T}}\left(-\log \mathcal{D}_{\mathrm{T}}\right)\right)_{\mathrm{S}_{t}}\right.$ with Kodaira-Spencer classes. Note that

$$
\left.p_{\mathrm{T}}^{*} \Theta_{\mathrm{T}}\left(-\log \mathcal{D}_{\mathrm{T}}\right)\right|_{\mathrm{S}_{t}} \simeq \mathcal{O}_{\mathrm{S}_{t}} \text { and }\left.p_{\mathrm{S}}^{*} \Theta_{\mathrm{S}}\left(-\log \mathcal{D}_{\mathrm{S}}\right)\right|_{\mathrm{T}_{j}} \simeq \mathcal{O}_{\mathrm{T}_{j}} .
$$

Let $\left.1_{T} \in p_{\mathrm{T}}^{*} \Theta_{\mathrm{T}}\left(-\log \mathcal{D}_{\mathrm{T}}\right)\right|_{\mathrm{S}_{i}}$ and $\left.1_{S} \in p_{\mathrm{S}}^{*} \Theta_{\mathrm{S}}\left(-\log \mathcal{D}_{\mathrm{S}}\right)\right|_{\mathrm{T}_{j}}$ be the constant section. Then by using a Theorem of Jost and Yau proved in [20] one obtains endomorphisms

$$
\left.\theta\right|_{S_{t}}\left(1_{\mathrm{S} t}\right): \mathbb{V}_{\mathrm{S}_{i}^{0}} \rightarrow \mathbb{V}_{\mathrm{S}_{i}^{0}} \text { and }\left.\theta\right|_{\mathrm{T}_{j}}\left(1_{\mathrm{T}_{t}}\right): \mathbb{V}_{\mathrm{T}_{j}^{0}} \rightarrow \mathbb{V}_{\mathrm{T}_{j}^{0}}
$$

See also [51] for another proof. This proof is based on the observation that the image of the map (38) is contained in the kernel of the induced Higgs map on $\operatorname{End}(\mathbb{V})$. Therefore, the image is a Higgs subsheaf with the trivial Higgs field. The Higgs poly-stability of $\left.\operatorname{End}(\mathbb{V})\right|_{S_{t}}$ implies that any section in this subsheaf is flat. One sees also that this flat section is of Hodge type $(-1,1)$. 
2. Let $\mathbb{V}$ be an arbitrary Variation of Hodge Structure over some quasiprojective space by taking the local monodromy by the following consideration. Let $\mathrm{S}^{0}=\mathrm{S} \backslash\left\{s_{0},,, s_{m}\right\}$ and $\mathrm{T}^{0}=\mathrm{T} \backslash\left\{t_{0}, \ldots, t_{n}\right\}$ be two smooth projective curves. The local system $\mathbb{V}$ corresponds to a representation

$$
\rho: \pi_{1}\left(\mathrm{~S}^{0}, *\right) \times \pi_{1}\left(\mathrm{~T}^{0}, *\right) \rightarrow G L(V),
$$

and the restriction $\left.\rho\right|_{\mathrm{S}_{t}}$ corresponds to the restricted representation $\left.\rho\right|_{\pi_{1}\left(\mathrm{~S}^{0}, *\right)}$ : $\pi_{1}\left(\mathrm{~S}^{0}, *\right) \rightarrow G L(V)$. Let $\gamma_{t_{j}}$ be a short loop around some point $t_{j}$, then it commutes with $\pi_{1}\left(\mathrm{~S}^{0}, *\right)$. Hence, $\rho\left(\gamma_{t}\right)$ descends to an endomorphism, $\operatorname{say} \rho\left(\gamma_{t_{j}}\right): \mathbb{V}_{S_{t}^{0}} \rightarrow \mathbb{V}_{S_{t}^{0}}$. Similarly, one gets also an endomorphism $\rho\left(\gamma_{s_{i}}\right)$ on $\mathbb{V}_{\mathrm{T}_{s}} \rightarrow \mathbb{V}_{\mathrm{T}_{s}}$. It is well known that those two endomorphisms $\rho\left(\gamma_{t_{j}}\right)$ and $\rho\left(\gamma_{s_{i}}\right)$ are unipotent. Let $\mathcal{N}_{s_{i}}=\log \left(\mathcal{T}_{i}-i d\right)$ and $\mathcal{N}_{t_{j}}=\log \left(\mathcal{T}_{j}-i d\right)$ be the nilpotent part of $\mathcal{T}_{i}$ and $\mathcal{T}_{j}$ respectively which are non-trivial by assumption. Let $\left.\theta\right|_{S_{t}}\left(1_{\mathrm{S}_{t}}\right): \mathbb{V}_{\mathrm{S}_{i}^{0}} \rightarrow \mathbb{V}_{\mathrm{S}_{i}^{0}}$ be the endomorphism of the variations of the Hodge structure induced by $\left.\theta\right|_{S_{t}}\left(1_{\mathrm{S} t}\right)$ in (39). We noticed that $\left.\theta\right|_{S_{t}}\left(1_{\mathrm{S} t}\right)$ and $\left.\theta\right|_{T_{j}}\left(1_{\mathrm{T} s}\right)$ are nilpotent. Similar to what Schmid did for nilpotent endomorphisms on vector space we see that $\left.\theta\right|_{S_{t}}\left(1_{\mathrm{St}}\right)$ induces a weight filtration of the local system $0 \subset \mathbb{W}_{0} \subset \mathbb{W}_{1} \subset \ldots \mathbb{W}_{2 k-1} \subset \mathbb{W}_{2 k}=\mathbb{V}_{\mathrm{S}_{t_{0}}^{0}}$, such that $\left.\theta\right|_{S_{t}}\left(1_{S t}\right)\left(\mathbb{W}_{l}\right) \subset \mathbb{W}_{l-2}$. Since the local system $\mathbb{V}_{S_{t_{0}}^{0}}$ is completely reducible

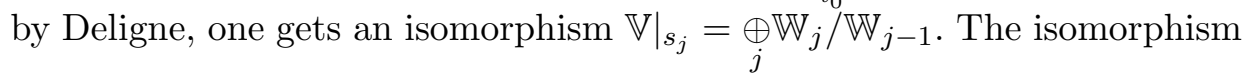
$\left.\theta\right|_{S_{t}}\left(1_{\mathrm{St}}\right)$ shifts $\mathbb{W}_{l} / \mathbb{W}_{l-1}$ to $\bigoplus_{j<l-2} \mathbb{W}_{j} / \mathbb{W}_{j-1}$. On the other hand, regarding $\mathcal{T}_{i}$ as an element in the image $\rho: \pi_{1}\left(\mathrm{~S}_{t_{0}}, s_{0}\right) \rightarrow G L(V)$ the above decomposition on the fibre $\mathbb{V}_{s_{0}, t_{0}}$ decomposes $\mathcal{N}_{s_{i}}=\log \left(\mathcal{T}_{i}-i d\right)$ as a direct sum of endomorphisms on $\left(\mathbb{W}_{j}\right)_{\left(s_{0}, t_{0}\right)} /\left(\mathbb{W}_{j-1}\right)_{\left(s_{0}, t_{0}\right)}$. In particular, $\mathcal{N}_{s_{i}}$ can not be equal to $\mathcal{N}_{t_{j}}$ as endomorphism on the fibre $\mathbb{V}_{\left(s_{0}, t_{0}\right)}$ since the filtrations of those two parallel endomorphisms of VHS will be different since the families obtained from the restrictions of the family (37) on $S$ and $T$ are not isomorphic. This follows from local Torelli Theorem. The two filtrations will be invariant under the actions of $\log \left(\mathcal{T}_{i}-i d\right)$ and $\log \left(\mathcal{T}_{j}-i d\right)$ respectively. Theorem 45 is proved.

Corollary 46 Suppose that $S^{0}$ and $T^{0}$ are Zariski open sets in projective varieties $S$ and $T$. Suppose that $\pi: \mathcal{X} \rightarrow \mathcal{Y}=S \times T$ is the family of Calabi-Yau manifolds (37), such that the induced map to the moduli space $\overline{\mathcal{M}_{L}(M)}$ is generically finite. Suppose that the discriminant locus $\mathfrak{D}_{\infty}=\overline{\mathcal{M}_{L}(M)} \ominus \mathcal{M}_{L}(M)$ is a irreducible divisor and at some point $\left(s_{0}, t_{0}\right)$ of $\mathfrak{D}_{\infty}=\left(S \ominus S^{0}\right) \times\left(T \ominus T^{0}\right)$ the local monodromy operators of the restrictions of the family (37) on $S$ and $T$ around the points $s_{0} \in S$ and $t_{0} \in T$ are infinite. Then the family (37) is rigid. 
Proof: Since we assumed that the discriminant locus $\mathfrak{D}_{\infty}=\overline{\mathcal{M}_{L}(\mathrm{M})} \ominus$ $\mathcal{M}_{L}(\mathrm{M})$ is an irreducible divisor in $\overline{\mathcal{M}_{L}(\mathrm{M})}$ then the monodromy operators acting on the middle cohomology of a fixed CY manifold induced by any loops $\gamma$ in $\mathcal{M}_{L}(\mathrm{M})$ around the image of the point $\left(s_{0}, t_{0}\right)$ in $\mathfrak{D}_{\infty}$ will be the same. Since we assumed that the monodromy operators are infinite we get a contradiction with Theorem 45 .

Remark 47 It is a well known fact that the discriminant locus $\mathfrak{D}_{\infty}$ in the Hilbert scheme of hypersurfaces in $\mathbb{C P}^{n}$ for $n \geq 2$ is an irreducible divisor.

The motivation of the next Theorem is based on a question raised by S.-T. Yau and this question is closely related to the example constructed in Appendix I. We will use the following definitions in the formulation of Theorem 48: We will say that $\mathbb{V}$ is a K3 like, abelian or elliptic local system it is a VHS of weight two induced by an Euclidean lattice with signature $(2, k)$ where $0<k \leq 19$, or a VHS of an abelian variety or elliptic curve respectively.

Theorem 48 Suppose that

$$
\pi: \mathcal{X} \rightarrow S^{0} \times T^{0}=\mathcal{Y}_{0}
$$

is a family of Calabi-Yau manifolds, such that the induced map into the moduli space is generically finite where $S^{0}$ and $T^{0}$ are Zariski open sets in projective varieties $S$ and $T$. Suppose that $\mathbb{V} \rightarrow S^{0} \times T^{0}=\mathcal{Y}_{0}$ is the VHS induced by the family (37). Fixing $t \in T^{0}$, then the local system $\left.\mathbb{V} \otimes \mathbb{C}\right|_{S_{t}}$ has a splitting over $\mathbb{C}$ and the splittings of the local systems are either

$$
\mathbb{V} \otimes \mathbb{C}_{S_{t}}=\mathbb{G}_{0} \oplus \mathbb{G}_{1} \oplus \mathbb{G}_{2}
$$

where $\mathbb{G}_{0}$ and $\mathbb{G}_{2}$ are $K 3$ liked local systems which are isomorphic to each other and $\mathbb{G}_{1}$ is unitary local system or

$$
\mathbb{V} \otimes \mathbb{C}_{S_{t}}=\mathbb{G}_{0} \oplus \mathbb{G}_{1} \oplus \mathbb{G}_{2} \oplus \mathbb{G}_{3} \oplus \mathbb{G}_{4}
$$

where $\mathbb{G}_{0}$ and $\mathbb{G}_{4}$ are isomorphic elliptic-like local systems, $\mathbb{G}_{2}$ is an abelianlike local system and $\mathbb{G}_{1}$ and $\mathbb{G}_{3}$ are unitary local systems that are isomorphic to each other.

Proof: Consider the endomorphism $\sigma:\left.\left.\mathbb{V} \otimes \mathbb{C}\right|_{S_{t}^{0}} \rightarrow \mathbb{V} \otimes \mathbb{C}\right|_{S_{t}^{0}}$, which is the image of

$$
1_{S_{t}}:\left.\mathcal{O}_{\mathrm{S}_{t}} \rightarrow p_{\mathrm{T}}^{*} \Theta_{\mathrm{T}}\left(-\log \mathcal{D}_{\mathrm{T}}\right)\right|_{\mathrm{S}_{t}} \rightarrow \operatorname{End}\left(\left.\mathbb{V}\right|_{S_{t}}\right)
$$


Since we assumed that the family (40) is non-rigid, we have that $\sigma^{3}=0$. Otherwise if $\sigma^{3} \neq 0$ then by Theorem 38 the family (40) will be rigid. Clearly $\sigma$ is of $(-1,1)$ type. We know from Lemma 26 that any non rigid deformation corresponds to a parallel section of the pullback of the tangent bundle of the moduli space. Thus this parallel section can be identified with some Kodaira-Spencer class $\theta$. Applying Cor. 6.5 in [43] to non-rigid families of CY 3 -folds, the three times iterated Kodaira-Spencer map

$$
\left(i_{T_{s}}^{*} \theta\right)^{3}:\left.\mathbb{V}\right|_{T_{s}} \rightarrow \mathbb{V}_{T_{s}} \otimes S^{3} \Omega_{T_{s}}^{1}\left(\log D_{T_{s}}\right), \quad \forall s \in S^{0}
$$

must be zero. Fixing $t \in T$, and varying $s$, the restriction $\left.\left(i_{T_{s}}^{*} \theta\right)\right|_{t, s}$ is precisely $\sigma$. Next we will consider two different cases:

Case 1. Suppose that $\sigma^{2}=0$. We consider the weight filtration studied in $[36]$ defined by $\sigma: \mathbb{W}_{0} \subset \mathbb{W}_{1} \subset \mathbb{W}_{2}=\left.\mathbb{V}\right|_{S_{t}}$. We set $\mathbb{W}_{0}=\sigma\left(\mathbb{W}_{2}\right), \quad \mathbb{W}_{1}=\operatorname{ker}\left(\sigma: \mathbb{W}_{2} \rightarrow \mathbb{W}_{0}\right)$. One has $\sigma: \mathbb{W}_{2} / \mathbb{W}_{1} \simeq \mathbb{W}_{0}$. Note that the Hodge bundle corresponding to $\mathbb{W}_{0}$ has the form

$$
\mathbb{W}_{0}=\sigma\left(E^{3,0}\right) \oplus \sigma\left(E^{2,1}\right) \oplus \sigma\left(E^{1,2}\right),
$$

setting $\mathbb{G}_{0}=\mathbb{W}_{0}, \mathbb{G}_{2}=\mathbb{W}_{2} / \mathbb{W}_{1}$ we obtain $\sigma: \mathbb{G}_{2} \simeq \mathbb{W}_{0}=\mathbb{G}_{0}$. Since from the definition of the mixed Hodge structure it follows that the pure Hodge structure on $\mathbb{W}_{2} / \mathbb{W}_{1}$ is of weight two then the VHS on $\mathbb{G}_{0}$ and $\mathbb{G}_{2}$ are K3 liked local system since the fibre of the family (40) is a CY manifold and $\operatorname{sodim}_{\mathbb{C}} \sigma\left(E^{3,0}\right)=1$. Note that the Hodge bundle corresponding to $\mathbb{W}_{1}$ has the form

$$
\operatorname{ker}\left(\sigma: E^{2,1} \rightarrow E^{1,2}\right) \oplus \operatorname{ker}\left(\sigma: E^{1,2} \rightarrow E^{0,3}\right) \oplus E^{0,3} .
$$

The Hodge bundle corresponding to $\mathbb{G}_{1}=\mathbb{W}_{1} / \mathbb{W}_{0}$ has the form

$$
\operatorname{ker}\left(\sigma: E^{2,1} \rightarrow E^{1,2}\right) / \sigma\left(E^{3,0}\right) \oplus \operatorname{ker}\left(\sigma: E^{1,2} \rightarrow E^{0,3}\right) / \sigma\left(E^{2,1}\right) .
$$

Since $\left.\mathbb{V}\right|_{S_{t}}$ is complete reducible by Deligne, we obtain $\left.\mathbb{V}\right|_{S_{t}} \simeq \bigoplus_{i=0}^{2} \mathbb{G}_{i}$.

Case 2) Suppose now $\sigma^{2} \neq 0, \sigma^{3}=0$. Consider again the weight filtration

$$
0 \subset \mathbb{W}_{0} \subset \mathbb{W}_{1} \subset \mathbb{W}_{2} \subset \mathbb{W}_{3} \subset \mathbb{W}_{4}=\left.\mathbb{V}\right|_{S_{t}}
$$

Set $\mathbb{W}_{0}:=\sigma^{2}\left(\mathbb{W}_{4}\right), \quad \mathbb{W}_{3}:=\operatorname{ker}\left(\sigma^{2}: \mathbb{W}_{4} \rightarrow \mathbb{W}_{0}\right)$ Note that $\sigma$ is of $(-1,1)$ type, so the only possible non-zero parts of $\sigma^{2}$ are

$$
\sigma^{2}\left(E^{2,1}\right) \subseteq E^{0,3}, \quad \sigma^{2}\left(E^{3,0}\right) \subset E^{1,2}
$$


they are dual to each other. Thus, if $\sigma^{2} \neq 0$, then

$$
\sigma^{2}\left(E^{2,1}\right)=E^{0,3} \text { and } \sigma^{2}\left(E^{3,0}\right) \subset E^{1,2} .
$$

Therefore, $\sigma^{2}: \mathbb{W}_{4} / \mathbb{W}_{3} \simeq \mathbb{W}_{0}$ And the Hodge bundle corresponding to $\mathbb{W}_{0}$ has the form $\theta: F_{0}^{1,0} \rightarrow F_{0}^{0,1}$, where $F_{0}^{1,0}=\sigma^{2}\left(E^{3,0}\right), F_{0}^{0,1}=E^{0,3}$ and $\theta$ is the original Kodaira-Spencer class restricted to $\bigoplus_{p+q=1} F_{0}^{p, q} \subset \bigoplus_{p+q=3} E^{p, q}$. Thus the VHS on $\mathbb{W}_{0}$ is like the VHS on an elliptic curve. We set now $\mathbb{W}_{3}=\operatorname{ker}\left(\sigma^{2}: \mathbb{W}_{4} \rightarrow \mathbb{W}_{0}\right)$ whose Hodge bundle has the form

$$
\mathbb{W}_{3}=\operatorname{ker}\left(\sigma^{2}: E^{2,1} \rightarrow E^{0,3}\right) \oplus E^{1,2} \oplus E^{0,3} .
$$

Let $\mathbb{W}_{1}=\sigma\left(\mathbb{W}_{3}\right)$. Then $\mathbb{W}_{1}$ as a Hodge bundle has the form

$\mathbb{W}_{1}=\sigma\left(\operatorname{ker}\left(\sigma^{2}: E^{2,1} \rightarrow E^{0,3}\right)\right) \oplus \sigma\left(E^{1,2}\right)=\sigma\left(\operatorname{ker}\left(\sigma^{2}: E^{2,1} \rightarrow E^{0,3}\right)\right) \oplus E^{0,3}$

Let $\mathbb{W}_{2}=\left\{v \in \mid \sigma(v) \in \mathbb{W}_{0}\right\}$. Clearly we have $\sigma: \mathbb{W}_{3} / \mathbb{W}_{2} \simeq \mathbb{W}_{1} / \mathbb{W}_{0}$. Remember that the Hodge bundle corresponding to $\mathbb{W}_{0}$ has weight one and thus the form $\sigma^{2}\left(E^{3,0}\right) \oplus E^{0,3}$. The Hodge bundle corresponding to $\mathbb{W}_{1} / \mathbb{W}_{0}$ has the form

$$
F^{0,0}=\sigma\left(\operatorname{ker}\left(\sigma^{2}: E^{2,1} \rightarrow E^{0,3}\right) / \sigma^{2}\left(E^{3,0}\right) .\right.
$$

So, we see that $\sigma: \mathbb{W}_{3} / \mathbb{W}_{2} \simeq \mathbb{W}_{1} / \mathbb{W}_{0}$ are unitary local systems. Finally we want to determine the Hodge bundle corresponding to $\mathbb{W}_{2}$. It is just the preimage of the Hodge bundle corresponding to $\mathbb{W}_{0}$, namely

$$
\sigma^{-1}\left(\sigma^{2}\left(E^{3,0}\right)\right) \cap E^{2,1} \oplus E^{1,2} \oplus E^{0,3} .
$$

So, the Hodge bundle corresponding to $\mathbb{W}_{2} / \mathbb{W}_{1}$ has the form

$$
\sigma^{-1}\left(\sigma^{2}\left(E^{3,0}\right)\right) \cap E^{2,1} \oplus E^{1,2} / \sigma\left(\operatorname{ker}\left(\sigma^{2}: E^{2,1} \rightarrow E^{0,3}\right)\right) .
$$

It is easy to see that it is again abelian variety liked local system. Theorem 48 is proved.

\section{Appendix I. Complex Exponential Maps in Kähler Geometry}

It is stated in [16] that the following conditions are equivalent: $\mathbf{1} \cdot g_{i, \bar{j}}$ is a Kähler metric on a complex manifold M. 2. The $(1,1)$ form $\omega:=\operatorname{Im}(g)$ is 
closed. 3. The complex structure operator $J_{M}$ on $\mathrm{M}$ is a parallel tensor with respect to the Levi-Cevita connection, i.e. $\nabla J_{\mathrm{M}}=04$. Around any point $m_{0} \in M$ there exist holomorphic coordinates $\left\{z^{1}, \ldots, z^{n}\right\}$ in an open set $\mathcal{U} \subset M$ such that locally the metric $g$ is given by:

$$
g_{i, \bar{j}}=\delta_{i j}+\frac{1}{4} \sum_{k, l} R_{i \bar{j}, k l} z^{k} \overline{z^{l}}+\ldots
$$

where $R_{i \bar{j}, k \bar{l}}$ is the curvature tensor.

The coordinates $\left\{z^{1}, \ldots, z^{n}\right\}$ will be called flat coordinates with respect to the Kähler metric g. Let us fix a point $m_{0}$ in the complex Kähler manifold M. Let $T_{m_{0}}$ be the tangent space at the point $m_{0} \in M$. We will consider for the moment the tangent space $T_{m_{0}}$ as a real $2 \mathrm{n}$ dimensional vector space. Let $e_{1} \in T_{m_{0}}$ be a vector of length 1 . We will define $e_{n+1}:=J_{\mathrm{M}} e_{1}$. Let $e_{2}$ be a vector perpendicular to the vectors $e_{1}$ and $e_{n+1}$ and $\left\|e_{2}\right\|=1$. We will define $e_{n+2}:=J_{\mathrm{M}} e_{2}$. By continuing this process we obtain a basis in $T_{m_{0}}$ consisting of vectors $\left\{e_{1}, . ., e_{n}, J_{\mathrm{M}} e_{1}=e_{n+1}, \ldots, J_{\mathrm{M}} e_{n}=e_{2 n}\right\}$ such that they satisfy $\left\langle e_{i}, e_{j}\right\rangle=\delta_{i j}$ Let $\gamma_{i}(t)$ be geodesics for $|t|<\varepsilon$ with respect to the metric $\mathrm{g}$ on $\mathrm{M}$ such that $\gamma_{i}(0)=m_{0} \in \mathrm{M}$ and $\left.\frac{d \gamma_{i}(t)}{d t}\right|_{t=0}=e_{i}$ for $i=1, . ., n$.

Definition 49 We will define two dimensional distributions $\mathcal{D}_{i}$ in the tangent bundle $T(M)$ in a small neighborhood $\mathcal{U}$ of the point $m_{0} \in M$. $\mathcal{D}_{i}$ by parallel transport along the geodesics containing the point $m_{0}$ of the two dimensional subspaces $\mathcal{E}_{i}(0) \subset T_{m_{0}, M}$ span by $e_{i}$ and $J_{M} e_{i}$ for $i=1, \ldots, n$.

The following theorem is probably well known. We will outline its proof only.

Theorem 50 Locally around the point $m_{0} \in M$ there exist one dimensional complex manifolds $Z_{i}$ for each $1 \leq i \leq n$ such that 1 . For each point $x \in$ $\gamma_{i}(t) \subset Z_{i}$ the tangent space $T_{x, Z_{i}}$ of $Z_{i}$ is equal to $\mathcal{D}_{i}(t)$. 2. $Z_{i}$ is a totally geodesic two dimensional real submanifold.

Proof: We will construct $Z_{i}$ for $i=1, \ldots, n$ based on the following Proposition:

Proposition 51 The distributions $\mathcal{D}_{i}$ are integrable distributions.

Proof: The proof of Proposition 51 follows almost directly from the definition of the integrability.

Proposition 52 There exists a unique totally geodesic two dimensional real submanifold $Z_{i}$ such that at each point $m \in Z_{i}$ we have: $T_{m, Z_{i}}=\left.\mathcal{D}_{i}\right|_{m}$. 
Proof: The proof is obvious.

Proposition 52 implies Theorem 50.

Remark 53 Theorem 50 implies that to the pairs $\left(e_{i}, J_{M} e_{i}\right)$ of vectors in $T_{m_{0}, M_{0}}$ we can associate a system of local holomorphic coordinates $\left(z^{1}, \ldots, z^{n}\right)$ in a small neighborhood of $m_{0} \in M_{0}$. The complex curves $Z_{i}$ are defined by the system of equations: $z^{1}=\ldots=z^{i-1}=z^{i+1}=\ldots=z^{n}=0$.

Theorem 54 Let $g$ be a Kähler metric on M. Let $\left(z^{1}, \ldots, z^{n}\right)$ be the coordinate system as defined in Remark 53 in a small neighborhood of $m_{0} \in M$. Then we have the following expression for $g_{i, \bar{j}}$ :

$$
g_{i, \bar{j}}(z, \bar{z})=\delta_{i, \bar{j}}+\frac{1}{6} R_{i, \bar{j}, k, \bar{l}} z^{k} \bar{z}^{l}+\ldots
$$

where $R_{i, \bar{j}, k, \bar{l}}$ is the curvature tensor.

Proof: Let us consider the geodesic coordinates $\left(x^{1}, y^{1} ; \ldots, x^{n}, y^{n}\right)$ associated to the orthonormal frame $\left(e_{1}, J_{\mathrm{M}} e_{1} ;,, ; e_{n}, J_{\mathrm{M}} e_{n}\right)$. In the geodesic coordinate system associated to $\left(e_{1}, J_{\mathrm{M}} e_{1} ;, \ldots ; e_{n}, J_{\mathrm{M}} e_{n}\right)$, we have:

$$
\left.\frac{\partial}{\partial x^{m}}\left(g_{i, j}\right)\right|_{m_{0}}=\left.\frac{\partial}{\partial y^{m}}\left(g_{i, j}\right)\right|_{m_{0}}=0
$$

for $m=1, \ldots, n$. This follows from the following Proposition:

Proposition 55 At the origin of a geodesic coordinate system, the metric has the following Taylor expansion:

$$
g_{i j}=\delta_{i j}+\frac{1}{3} R_{i p, g j} x^{p} x^{q}+O\left(|x|^{3}\right),
$$

where $R_{i, p, q, j}$ is the curvature tensor of $g$. (See Proposition 1.14 on page 8 of the book [32].)

On the other hand, Theorem 50 implies that we have the following relations between the coordinate systems $\left(x^{1}, y^{2} ; \ldots ; x^{n}, y^{n}\right)$ and $\left(z^{1}, \ldots, z^{n}\right)$ $z^{i}=x^{i}+\sqrt{-1} y^{i}$. So we deduce that $\left.\frac{\partial}{\partial z k}\left(g_{i, \bar{j}}\right)\right|_{m_{0}}=\left.\frac{\bar{\partial}}{\overline{\partial z^{i}}}\left(g_{i, \bar{j}}\right)\right|_{m_{0}}=0$. From here and Proposition 55 we can conclude that

$$
g_{i, \bar{j}}(z, \bar{z})=\delta_{i, \bar{j}}+\frac{1}{6} R_{i, \bar{j}, k, \bar{l}} z^{k} \bar{z}^{l}+\ldots
$$

where $R_{i, \bar{j}, k, \bar{l}}$ is the curvature tensor. This proves Theorem 54 . 
Corollary 56 Let $M$ be a Kähler Manifold with a Kähler metric $g_{i, \bar{j}}(z, \bar{z})$ such that $g_{i, \bar{j}}(z, \bar{z})$ are real analytic functions with respect to a local holomorphic coordinates, then we can identify the totally geodesic one complex dimensional manifolds $\gamma(\tau)$ in $M$ at a point $m \in M$ with respect to the Kähler metric $g_{i, \bar{j}}(z, \bar{z})$ with the complex lines $l \subset T_{m, M}=\mathbb{C}^{N}$ through the origin $0 \in \mathbb{C}^{N}$ with directions defined by $\left.\frac{d}{d \tau} \gamma(\tau)\right|_{0}$.

Definition 57 The map defined in Cor. 56 we will be called the complex exponential map in Kähler Geometry.

\section{Appendix II. Applications of Bishop's Conver- gence Theorem}

We will prove the following Theorem following the arguments in [34]:

Theorem 58 Let $\pi: \mathcal{N} \rightarrow \mathcal{K}$ and $\pi^{\prime}: \mathcal{N}^{\prime} \rightarrow \mathcal{K}$ be two holomorphic families of Kähler manifolds over a complx manifold $\mathcal{K}$ as a parameter space. Suppose that both families can be identified with the $C^{\infty}$ trivial family $\mathcal{K} \times N_{0}$ by some diffeomorphism $\phi$ isotopic to the identity. Let $0 \in \mathcal{K}$ be a fixed point. Let $\tau_{n} \in \mathcal{K}$ be a sequence of points in $\mathcal{K}$ such that

$$
\lim _{k \rightarrow \infty} \tau_{k}=\tau_{0}
$$

Suppose that for all $k$ there exist biholomorphic maps $\phi_{k}: N_{\tau_{k}} \rightarrow N_{\tau_{k}}^{\prime}$ such that $\phi_{k}$ induces the identity map on $H^{2}\left(N_{0}, \mathbb{Z}\right)$. Then $N_{\tau_{0}}$ and $N_{\tau_{0}}^{\prime}$ are bimeromorphic

Proof: For each $\tau_{k}$ we have a biholomorphic map $\phi_{k}: N_{\tau_{k}}^{\prime} \rightarrow N_{\tau_{k}}$ then the graphs of $\phi_{k}$ are submanifolds $\Gamma_{k} \subset N_{\tau_{k}}^{\prime} \times N_{\tau_{k}}$. The idea of the proof is to show that

$$
\lim _{k \rightarrow \infty} \Gamma_{k}=\Gamma_{0} \subset N_{\tau_{k}}^{\prime} \times N_{\tau_{0}}
$$

exists and from this fact to deduce Theorem 58 .

Lemma 59 There exists a subsequence $\left\{k_{n}\right\}$ of the sequence $\{k\}$ such that the limit of the currents $\left[\Gamma_{k_{n}}\right] \lim _{n \rightarrow \infty}\left[\Gamma_{k_{n}}\right]=\left[\Gamma_{0}\right]$ exists, where $\Gamma_{0}$ is a complex analytic subspace in $N_{\tau_{o}} \times N_{\tau_{0}}$.

Proof: The proof of Lemma 59 is based on the following Theorem of Bishop. See [8]: 
Theorem 60 Let $M$ be a complex analytic manifold and let $\left[N_{n}\right]$ be a sequence of complex analytic subspaces considered as currents in M. Suppose that $h$ is a Hermitian metric on $M$ such that vol $\left(N_{n}\right) \leq C$ then there exists a subsequence of current $\left[N_{n_{k}}\right]$ such that the limits of the currents $\lim _{k \rightarrow \infty}\left[N_{n_{k}}\right]=\left[N_{0}\right]$ exists and as a current $\left[N_{0}\right]$ is defined by a complex ana-

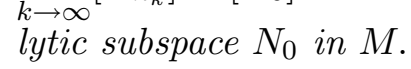

In order to apply Theorem 60 we need to construct a Hermitian metric $h$ on the complex manifold $\mathcal{M}=\mathcal{N}^{\prime} \underset{\mathcal{K}}{\times} \mathcal{N}$ and prove that the volume of the graphs $\Gamma_{k}$ of the isomorphisms $\phi_{k}$ are uniformly bounded

Construction of an Hermitian metric on $\mathcal{M}$. We assumed that $\mathcal{N} \rightarrow \mathcal{K}^{\prime}$ and $\mathcal{N} \rightarrow \mathcal{K}$ are families of Kähler manifolds. So we may we have two families of Kähler metrics $\omega^{1,1}(\tau)$ and $\omega_{1}^{1,1}(\tau)$ respectively defined on the fibres of $\mathcal{N}^{\prime}$ and $\mathcal{N}$ and the metrics depend on $\tau \in \mathcal{K}$ in a $C^{\infty}$ manner. Let

$$
\eta=\sqrt{-1} \sum_{i=1}^{N} d \tau^{i} \wedge \overline{d \tau^{i}}
$$

be a positive definite form on $\mathcal{U}$. The collection of forms $\omega^{1,1}(\tau), \omega_{1}^{1,1}(\tau)$ and $\eta$ define a Hermitian metric $H$ on $\mathcal{M}=\mathcal{N}^{\prime} \underset{\mathcal{K}}{\times} \mathcal{N}$.

Proposition 61 The submanifolds $\Gamma_{k}$ defined by (43) have a constant volume with respect to the family of metrics $\omega^{1,1}(\tau)+\omega_{1}^{1,1}(\tau)$ on $N_{\tau} \times N_{1, \tau}$, i.e. $\operatorname{vol}\left(\Gamma_{k}\right)=c$ for all $k$.

Proof: It is easy to see that

$$
\operatorname{vol}\left(\Gamma_{k}\right)=\int_{N_{k}^{\prime}}\left(\phi_{k}^{*}\left(\omega_{1}^{1,1}\left(\tau_{k}\right)+\omega^{1,1}\left(\tau_{k}\right)\right)^{2 n} .\right.
$$

Remember that $\phi_{k}^{*}$ is the identity map on $H^{2}(N, \mathbb{Z})$. Here we are using the fact that as $C^{\infty}$ manifolds $\mathcal{N}=\mathcal{K} \times N_{0}$ and $\mathcal{N}^{\prime}=\mathcal{K}^{\prime} \times N_{0}$ are diffeomorphic and the diffeomorphism which acts fibrewise is isotopic to the identity on $N_{0}$. Using this fact we see that the classes of cohomology of $\phi_{k}^{*}\left(\omega_{1}^{1,1}\left(\tau_{k}\right)\right)$ and $\omega^{1,1}\left(\tau_{k}\right)$ are fixed. Let us denote them by $\left[\omega_{1}\right]$ and $[\omega]$ respectively. So we get that

$$
\int_{N_{k}^{\prime}}\left(\phi_{k}^{*}\left(\omega_{1}^{1,1}\left(\tau_{k}\right)\right)+\omega^{1,1}\left(\tau_{k}\right)\right)^{2 n}=\int_{N_{k}^{\prime}}\left(\left[\omega_{1}\right]+[\omega]\right)^{2 n}=c_{0} .
$$

Proposition 61 is proved. 
The end of the proof of Lemma 59: For a subvariety $Z$ of pure codimension $\mathrm{m}$ in a complex manifold $N_{\tau_{0}}$, we denote by $[Z]$ the current on $N_{\tau_{0}}$ defined by $Z$. Now we can apply Bishop's Theorem 60 and conclude that the sequence of currents converges weakly to a current $\left[\Gamma_{0}\right]$ in $N_{\tau_{0}} \times N_{\tau_{0}}^{\prime}$ of the form

$$
\left[\Gamma_{0}\right]=\sum_{i} m_{i}\left[\Gamma_{i}\right]
$$

where $m_{i}$ are positive integers and $\Gamma_{i}$ are irreducible complex analytic subspaces in $N_{\tau_{0}} \times N_{\tau_{0}}^{\prime}$. Lemma 59 is proved

The end of the proof of Theorem 58: Any closed current $[Z]$ on $N_{\tau_{0}} \times N_{\tau_{0}}^{\prime}$ defines a linear map:

$$
[Z]_{*}: H^{*}\left(N_{\tau_{0}}^{\prime}, \mathbb{C}\right) \rightarrow H^{*}\left(N_{\tau_{0}}, \mathbb{C}\right)
$$

between the cohomology rings of $N_{\tau_{0}}^{\prime}$ and $N_{\tau_{0}}$ as follows: Let $\alpha \in H^{*}\left(N_{\tau_{0}}^{\prime}, \mathbb{C}\right)$, then

$$
[Z]_{*}(\alpha):=\left(p r_{2}\right)_{*}\left([Z] \wedge\left(p r_{1}\right)^{*} \alpha\right)
$$

where $p r_{i}$ are respectively the projections of $N_{\tau_{0}} \times N_{0}^{\prime}$ onto the first and the second factor and $\left(p r_{1}\right)_{*}$ and $\left(p r_{2}\right)^{*}$ are the pushforward and the pullback maps. Similarly we define a linear map

$$
[Z]^{*}: H^{*}\left(N_{\tau_{0}}, \mathbb{C}\right) \rightarrow H^{*}\left(N_{\tau_{0}}^{\prime}, \mathbb{C}\right) .
$$

The map $\left[\Gamma_{0}\right]_{*}$ defined by the current $\left[\Gamma_{0}\right]$ clearly agrees with the map

$$
\lim _{k \rightarrow \infty}\left[\Gamma_{k}\right]=\lim _{k \rightarrow \infty} i d=i d=\left[\Gamma_{0}\right]_{*}
$$

on $H^{2 n}(N, \mathbb{C})$. From here we deduce that $\left[\Gamma_{0}\right]_{*}$ is the identity map on $H^{2 n}(N, \mathbb{C})$. This implies

$$
\left[\Gamma_{0}\right]_{*}\left(\wedge^{n}\left[\omega_{1}^{1,1}\right]\right)=\left(p r_{2}\right)_{*}\left(\sum_{i} m_{i}\left[\Gamma_{i}\right] \wedge\left(p r_{1}\right)^{*}\left(\wedge^{n}\left[\omega_{1}^{1,1}\right]\right)\right)=\wedge^{n}\left[\omega_{1}^{1,1}\right] .
$$

Hence there must be some $\Gamma_{j}$ among the components of which is projected both onto $N_{0}^{\prime}$ and $N_{\tau_{0}}$. There can be only one $\Gamma_{j}$ in $\Gamma_{0}$ with multiplicity $m_{j}=1$, because both

$$
\left(\sum_{i} m_{i}\left[\Gamma_{i}\right]\right)_{*} \text { and }\left(\sum_{i} m_{i}\left[\Gamma_{i}\right]\right)^{*}
$$

must leave fixed the class in $H^{0}(N, \mathbb{C})$ defined by the function 1 . This implies that the projection maps from $\Gamma_{j}$ to $N_{\tau_{0}}$ and to $N_{\tau_{0}}^{\prime}$ have degree one. So $N_{\tau_{0}}$ and $N_{\tau_{0}}^{\prime}$ are bimeromorphically equivalent. Theorem 58 is proved. 
Theorem 62 Suppose that the map $f: N_{\tau_{0}} \rightarrow N_{\tau_{0}}^{\prime}$ is bimeromorphic. Let $\mathcal{L}_{1}$ be an ample line bundle on $N_{\tau_{0}}^{\prime}$ such that $f^{*}\left(\mathcal{L}_{1}\right)$ is also an ample line bundle on $N_{\tau_{0}}$. Then $f$ is a biholomorphic map.

Proof: Since $f: N_{\tau_{0}} \rightarrow N_{\tau_{0}}^{\prime}$ is a bimeromorphic map, there exist complex analytic subspaces $Z_{1} \subset N_{\tau_{0}}$ and $Z_{2} \subset N_{\tau_{0}}^{\prime}$ of codim $\geq 2$ such that $f: N_{\tau_{0}} \ominus Z_{1} \rightarrow N_{\tau_{0}}^{\prime} \ominus Z_{2}$ is a biholomorphic map between those two Zariski open sets. From here it follows that $f^{*}\left(\mathcal{L}_{1}\right)$ is a well defined line bundle on $U:=N_{\tau_{0}} \ominus Z_{1}$. Since $\mathcal{L}_{1}$ is an ample bundle on $N_{\tau_{0}}^{\prime}$, $\left(\mathcal{L}_{1}\right)^{\otimes m}$ will be a very ample bundle on $N_{\tau_{0}}^{\prime}$ for some positive integer $m$. Let $\sigma_{0}, \ldots, \sigma_{k} \in H^{0}\left(N_{\tau_{0}}^{\prime},\left(\mathcal{L}_{1}\right)^{\otimes m}\right)$ be a basis in $H^{0}\left(N_{\tau_{0}}^{\prime},\left(\mathcal{L}_{1}\right)^{\otimes m}\right)$. Since $\left(\mathcal{L}_{1}\right)^{\otimes m}$ is a very ample line bundle then $\sigma_{0}, \ldots, \sigma_{k}$ define an embedding of $N_{\tau_{0}}^{\prime}$ into $\mathbb{P}^{k}$. Clearly $f^{*}\left(\sigma_{0}\right), . ., f^{*}\left(\sigma_{k}\right)$ will define an embedding of the Zariski open set $U_{0}:=N_{\tau_{0}} \ominus Z_{1}$ in $N_{\tau_{0}}$ into $\mathbb{P}^{k}$. Since $Z_{1}$ has a complex codimension $\geq 2$ Hartogs Theorem implies that the sections $f^{*}\left(\sigma_{0}\right), \ldots, f^{*}\left(\sigma_{k}\right)$ of the line bundle $f^{*}\left(\mathcal{L}_{1}\right)^{\otimes m}$ are well defined holomorphic sections on $N_{\tau_{0}}$ We assumed that $f^{*}\left(\mathcal{L}_{1}\right)$ is an ample line bundle on $N_{\tau_{0}}$ so we can choose $m$ to be large enough to conclude that $f^{*}\left(\mathcal{L}_{1}\right)^{\otimes m}$ is a very ample line bundle on $N_{\tau_{0}}$. From here we conclude that

$$
x \in N_{\tau_{0}} \rightarrow\left(f^{*}\left(\sigma_{0}\right)(x), \ldots, f^{*}\left(\sigma_{k}\right)(x)\right)
$$

defines a holomorphic embedding $\varphi: N_{\tau_{0}} \subset \mathbb{P}^{k}$. So we deduce that $f$ : $N_{\tau_{0}} \rightarrow N_{\tau_{0}}^{\prime}$ is an isomorphism. Theorem 62 is proved.

Remark 63 The arguments that we used to prove Theorem 58 are similar to the arguments used by Siu in [34]. These arguments were suggested by Deligne to D. Burns and Rapoport.

\section{Appendix III. Counter Examples to the Ana- logue of Shafarevich Conjecture of CY Mani- folds}

Let us consider K3 surfaces on which there exists an automorphism $\sigma$ of order two such that $\sigma$ has no fixed points. Such algebraic K3 surfaces exist and the quotient $X / \sigma=Y$ is an Enriques surface. It is a well known fact that $\sigma$ acts on the holomorphic two form $\omega_{X}$ on the K3 surface $X$ as follows: $\sigma^{*}\left(\omega_{X}\right)=-\omega_{X}$. Let us consider an elliptic curve $E_{\tau}:=\mathbb{C} / \Lambda_{\tau}$, where $\Lambda_{\tau}:=\{m+n \tau \mid m, n \in \mathbb{Z}, \tau \in \mathbb{C}$ and $\operatorname{Im} \tau>0\}$. We know that $E_{\tau}$ can be embedded in $\mathbb{C P}^{2}$ and in one of the standard affine open sets $E_{\tau}$ 
is given by the equation: $y^{2}=4 x^{3}+g_{2} x+g_{3}$, where $g_{2}(\tau)=60 E_{4}(\tau)$ and $g_{3}(\tau)=140 E_{6}(\tau) . E_{4}(\tau)$ and $E_{6}(\tau)$ are the Eisenstein series. Let $\sigma_{1}$ be the involution defined on the equation of the elliptic curve as follows: $\sigma_{1}(x, y)=(-x, y)$. Clearly $\sigma_{1}$ acts on $E_{\tau}$ and $E_{\tau} / \sigma_{1}=\mathbb{C P}^{1}$. Thus we obtain that $\sigma_{1}$ acts on the holomorphic one form $d z_{\tau}=\frac{d x}{y}$ on $E_{\tau}$ as follows: $\sigma_{1}^{*}\left(d z_{\tau}\right)=-d z_{\tau}$. From here it is very easy to see that the quotient of the product $X \times E_{\tau} / \sigma \times \sigma_{1}=\mathrm{M}$ will be a CY manifold. These CY manifolds are called Borcea-Voisin manifolds. From the theory of moduli of Enriques surfaces and of elliptic curves it follows that the moduli space $\mathcal{M}(\mathrm{M})$ of $\mathrm{M}$ is isomorphic to $\Gamma \backslash \mathfrak{h}_{2,10} \times \mathbb{P S L}_{2}(\mathbb{Z}) \backslash \mathfrak{h}$. For more detail about moduli of Enriques surfaces see [6]. Let $\mathcal{X} \rightarrow C$ be a non isotrivial family of K3 surfaces with an involution $\sigma$ without fixed points acting on the non singular fibres of the above family. In [18] it was proved that the set $\mathcal{D}_{C}$ of points in $C$ over which the fibres are singular is not empty. We will call the set $\mathcal{D}_{C}$ the discriminant locus.

Let $E_{\lambda}$ be the family of elliptic curves: $y^{2}=x(x-1)(x-\lambda)$. Clearly we have defined a family of elliptic curves over the projective line. Let us denote it by $\tilde{E} \rightarrow \mathbb{C P}^{1}$. Let us take the product

$$
\mathcal{X} \times \tilde{E} \rightarrow C \times \mathbb{C P}^{1} .
$$

On the family (44) we can define the action of $\sigma$ and $\sigma_{1}$. By taking the quotient we will get a family

$$
\mathcal{Y} \rightarrow C \times \mathbb{C P}^{1} .
$$

of three dimensional CY manifolds over the product space $C \times \mathbb{C P}^{1}$. Clearly that for fixed $\lambda \in \mathbb{C P}^{1}$ and $\lambda \neq 0,1 \& \infty$ we can identify all curves $C_{\lambda}=$ $C \times \lambda$. Moreover under this identification the discriminant locuses $\mathcal{D}_{C \lambda}$ are identified too. This means that the discriminant locus of the family (45) satisfies: $\mathcal{D}_{\mathbb{C P}^{1} \times C}=p_{1}^{*} \mathcal{D}_{\mathbb{C P}^{1}}+p_{2}^{*} \mathcal{D}_{C}$. So the family (45) is not rigid and gives a counter example to Shafarevich's conjecture.

\section{References}

[1] Y. Andre, "On Shafarevich and Tate Conjectures for Hyper-Kählerian Varieties", Math. Ann. 305(1996) 205-248.

[2] S. Yu. Arakelov, "Families of Algebraic Curves with Fixed Degeneracies", Izv. Akad. Nauk SSSR Ser. Mat. 35(1971).

[3] A. Borel, "Some Metric Properties of Arithmetic Quotients of Bounded Symmetric Domains", J. of Diff. Geom. 6(1972), 543-560. 
[4] W. Baily and Borel, "On Compactification of Arithmetically Defined Quotients of Bounded Symmetric Domains", Bull. Amer. Math. Society 70(1964) 588-593.

[5] W. Baily and Borel, "On Compactification of Arithmetic Quotients of Bounded Symmetric Domains", Ann. Math. (2) 84(1966) 442-528.

[6] W. Barth, C. Peters and A. Van de Ven, "Compact Complex Surfaces", Ergebnisse der Math. 4 New York, Springer-Verlag (1984).

[7] E. Bedulev and E. Viehweg, "On Shafarevich Conjecture for Surfaces of General Type over Function Fields", Inv. Math. 139(2000), 603-615, math.ALG/9904124.

[8] E. Bishop, "Conditions for the Analyticity of Certain Sets." Mich. Math. J. 11(1964) 289-304.

[9] J.-P. Demailly, " L ${ }^{2}$ Vanishing Theorems for Positive Line Bundles and Adjunction Theory", Lecture Notes in Mathematics vol. 1646, p. 1-97.

[10] S. Donaldson, "Polynomial Invariants for Smooth 4-Manifolds", Topology 29(1990), No. 3, 257-315.

[11] A. Grothendieck, "Éléments de Géométrie Algébrique", IHES, Publications Mathématiques, vol. 8 .

[12] A. Grothendieck, "Séminaire de Géométrie Algébrique", IHES, Paris, 1960-1961.

[13] G. Faltings, "Arakelov's Theorem for Abelian Varieties", Inv. Math. 73(1983) 337-347.

[14] Grauert, "Über Modifikationed und Exzeptionelle Mengen", Math. Ann. 146(1962), 331-368.

[15] P. Griffiths, "Periods of Integrals on Algebraic Manifolds", Publ.Math. IHES vol. 38(1970), 125-180.

[16] P. Griffiths and J. Harris, "Principle of Algebraic Geometry".

[17] J. Jorgenson and A. Todorov, "Analytic Discriminant for Manifolds with Zero Canonical Class", Manifolds and Geometry, ed. P. de Bartolomeis, F. Tircerri and E. Vesantini, Symposia Mathematica 36, (1996) 223-260. 
[18] J. Jorgenson and A. Todorov, "Enriques Surfaces, Analytic Discriminants, and Borcherds's $\Psi$ Function", Com. Math. Phys. 191(1998), 249-264.

[19] J. Jorgenson and A. Todorov, Correction to "Enriques Surfaces, Analytic Discriminants, and Borcherds's $\Psi$ Function", Com. Math. Phys..

[20] J. Jost and S. -T. Yau, "Harmonic Mappings and Algebraic Varieties Over Function Fields", Amer. J. Math. 115(1993) 1197-1227.

[21] S. Kovàcs, "Families Over Base with Birationally Nef Tangent Bundle", J. Reine Angew. Math. 487 (1997), 171-177.

[22] B. Lian, A. Todorov and S.-T. Yau, " Maximal Unipotent Elements in the Monodromy of Complete Intersections $C Y$ ", preprint.

[23] K. Liu," Geometric Height Inequalities", Math. Research Letter 3, 693702(1996).

[24] Z. Lu, "On the Curvature Thensor of the Hodge Metric of Moduli of Polarized Calabi-Yau Threefolds", J. Geom. Analysis, 11(2001), No 4, 633-645.

[25] Z. Lu and X. Sun, "Weil-Petersson Geometry on Moduli space of Polarized Calabi-Yau Manifolds." preprint.

[26] T. Matsusaka and D. Mumford, "Two Fundamental Theorems on Deformations of Polarized Varieties", Amer. J. Math. 86(1964).

[27] L. Migliorini, " A Smooth Family of Minimal Surfaces of General Type over a Curve of Genus at Most One is trivial" J. Alg. Geom. 4(1995) 353-361.

[28] R. Palais, "On the Existance of Slices for Actions of Non-Compact Lie Groups", Ann. Math. (2) 73 (1961), 295-323.

[29] A. Parshin, "Algebraic Curves over Function Fields", Izv. Akad. Nauk. SSSR 32(1968) 1191-1219.

[30] C. Peters," Rigidity for Variations of Hodge Structures and Arakelovtype Finiteness Theorems", Compositio Math. 75(1990) 113-126.

[31] I. I. Piatetski-Shapiro and I. R. Shafarevich, "A Torelli Theorem for Algebraic Surfaces of Type K3", USSR Izv. Ser. Math. 5(1971) 547588. 
[32] John Roe, "Elliptic Operators, Topology and Asymptotic Methods" Pitman Research Notes in Mathematics Series 179, Longman Scientific \& Technical, 1988.

[33] H. -M. Saito and S. Zucker, "Classification of Non-Rigid Families of K3 Surfaces and Finiteness Theorem of Arakelov Type", Math. Ann. 298(1993) 1-31.

[34] Y. T. Siu, "Every K3-surface is Kähler." Invent. Math. 73 (1983), 139-150.

[35] Y. T. Siu, "Effective Very Ampleness", Inv. Math. 73(1995), 251-265.

[36] W. Schmidt, "Variations of Hodge Structures", Inv. Math. 22(1973), 211-319.

[37] I. R. Shafarevich, "Collected Mathematical Papers", New York: Springer-Verlag (1989).

[38] D. Sullivan, "Infinitesimal Computations in Topology", Publ. Math. IHES, No 47 (1977), 269-331.

[39] G. Tian, "Smoothness of the Universal Deformation Space of CalabiYau Manifolds and its Petersson-Weil Metric", Math. Aspects of String Theory, ed. S.-T.Yau, World Scientific (1998), 629-346.

[40] A. Todorov, "The Weil-Petersson Geometry of Moduli Spaces of $S U(n \geq 3)$ (Calabi-Yau Manifolds) I", Comm. Math. Phys. 126 (1989), $325-346$.

[41] E. Viehweg, "Quasi-Projective Moduli for Polarized Manifolds", Ergebnisse der Mathematik und iher Grenzgebiete 3. Folge, Band 30, Springer-Verlag, 1991.

[42] E. Viehweg, K. Zuo, "On the Isotriviality of Families of Projective Manifolds over Curves. ", J. Algebraic Geom. 10(2001), No. 4, 781799.

[43] E. Viehweg, K. Zuo, "Base Spaces of Non-Isotrivial Families of Smooth Minimal Models", In: Complex Geometry (Collection of Papers dedicated to Hans Grauert), Springer Verlag (2002) 279-328.

[44] E. Viehweg, K. Zuo, "On The Brody Hyperbolicity of Moduli Spaces for Canonically Polarized Manifolds", Duke Mathematical Journal, 118 (2003) 103-150, 
[45] E. Viehweg, K. Zuo, "Dicreteness of Minimal Models of Kodaira Dimension Zero and Subvarieties in Moduli Stacks.", MathAG/0210310.

[46] E. Viehweg, K. Zuo, "Complex Multiplication, GriffithsYukawa Coupling and Rigidity for Families of Hypersurafces", MathAG/0307398.

[47] S.-T. Yau, " A Generalized Schwarz Lemma for Kähler Manifolds", A. J. Mathematics, vol. 100, Issue 1(1978), 197-203.

[48] Qi Zhang, "Holomorphic One form on Projective Manifolds", J. Alg. Geom. 6(1997) 777-787.

[49] Yi Zhang, "The Rigidity of Families of Projective Calabi-Yau Manifolds", preprint MathAG/0308034.

[50] Yi Zhang, "On Families of Calabi-Yau Manifolds", Ph. D. Thesis, Chinese Uninersity Hong Kong, 2003.

[51] Kang Zuo, "On the Negativity of Kernels of Kodaira-Spencer Maps on Hodge Bundles and Applications.", Asian Journal of Mathematics vol. 4.

Kefeng Liu

Center of Mathematical Science,Zhejiang University; UCLA, Department of Mathematics, Los Angeles, CA 90095

Andrey Todorov

UC, Department of Mathematics Santa Cruz, CA 95064 Institute of Mathematics Bulgarian Academy of Sciences

Shing-Tung Yau

Center of Mathematical Science, Zheijiang University and Harvard University, Department of Mathematics, Cambridge, MA 02138

Kang Zuo

Chinese University of Hong Kong, Department of Mathematics, Shatin, Hong Kong and Department of Mathematics, University Mainz, 55099 Mainz 